\title{
High-Lift System for a Supercritical Airfoil: Simplified By ACtive Flow Control
}

\author{
LaTunia Pack Melton, Norman W. Schaeffler† and John C. Lin ${ }^{\ddagger}$ \\ NASA Langley Research Center, Hampton, Virginia, United States of America
}

Active flow control wind tunnel experiments were conducted in the NASA Langley LowTurbulence Pressure Tunnel using a two-dimensional supercritical high-lift airfoil with a $15 \%$ chord hinged leading-edge flap and a $25 \%$ chord hinged trailing-edge flap. This paper focuses on the application of zero-net-mass-flux periodic excitation near the airfoil trailingedge flap shoulder at a Mach number of 0.1 and chord Reynolds numbers of $1.2 \times 10^{6}$ to $9 \times 10^{6}$ with leading- and trailing-edge flap deflections of $-25^{\circ}$ and $30^{\circ}$, respectively. The purpose of the investigation was to increase the zero-net-mass-flux options for controlling trailing edge flap separation by using a larger model than used on the low Reynolds number version of this model and to investigate the effect of flow control at higher Reynolds numbers. Static and dynamic surface pressures and wake pressures were acquired to determine the effects of flow control on airfoil performance. Active flow control was applied both upstream of the trailing edge flap and immediately downstream of the trailing edge flap shoulder and the effects of Reynolds number, excitation frequency and amplitude are presented. The excitations around the trailing edge flap are then combined to control trailing edge flap separation. The combination of two closely spaced actuators around the trailing-edge flap knee was shown to increase the lift produced by an individual actuator. The phase sensitivity between two closely spaced actuators seen at low Reynolds number is confirmed at higher Reynolds numbers. The momentum input required to completely control flow separation on the configuration was larger than that available from the actuators used.

\section{Nomenclature}

\begin{tabular}{|c|c|c|c|c|c|}
\hline$c$ & $=$ & $\begin{array}{l}\text { reference airfoil chord } \\
\left(\text { at } \delta_{L E}=\delta_{T E}=0^{\circ}\right)\end{array}$ & $C_{\mu}$ & $=$ & $\begin{array}{l}\text { momentum coefficient, } \\
\equiv \rho_{j} h U_{j}^{2} / c q\end{array}$ \\
\hline$C_{d p}$ & $=$ & pressure drag coefficient & $C_{D}$ & $=$ & total drag \\
\hline$C_{L}$ & $=$ & lift coefficient & $C_{L, \max }$ & $=$ & maximum lift coefficient \\
\hline$C_{p}$ & $=$ & $\begin{array}{l}\text { pressure coefficient, } \\
\equiv\left(P-P_{s}\right) / q\end{array}$ & $f$ & $=$ & excitation frequency, $\mathrm{Hz}$ \\
\hline$F^{+}$ & $=$ & $\begin{array}{l}\text { reduced frequency, } \\
\equiv f x_{s p} / U_{\infty}\end{array}$ & $h$ & $=$ & slot height \\
\hline$P$ & $=$ & pressure & $P_{s}$ & $=$ & static pressure \\
\hline$q$ & $=$ & $\begin{array}{l}\text { freestream dynamic } \\
\text { pressure, } \equiv 1 / 2 \rho U_{\infty}^{2}\end{array}$ & $R e_{c}$ & $=$ & $\begin{array}{l}\text { chord Reynolds number, } \\
\equiv U_{\infty} c / \nu\end{array}$ \\
\hline$U$ & $=$ & Velocity & $U_{j}$ & $=$ & RMS of Jet exit velocity \\
\hline$V_{R M S}$ & $=$ & RMS voltage & $\mathrm{x} / \mathrm{c}$ & $=$ & $\begin{array}{l}\text { normalized streamwise loca- } \\
\text { tion }\end{array}$ \\
\hline $\mathrm{y} / \mathrm{c}$ & $=$ & $\begin{array}{l}\text { normalized cross-stream loca- } \\
\text { tion }\end{array}$ & $x_{s p}$ & $=$ & $\begin{array}{l}\text { distance from actuator to } \\
\text { trailing edge }\end{array}$ \\
\hline
\end{tabular}

*Research Scientist, Flow Physics and Control Branch, MS 170, Senior Member AIAA

${ }^{\dagger}$ Research Scientist, Flow Physics and Control Branch, MS 170, Member AIAA

${ }^{\ddagger}$ Research Scientist, Flow Physics and Control Branch, MS 170, Associate Fellow AIAA 


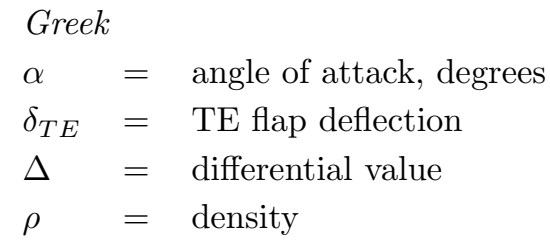

Abbreviations

$\begin{array}{ll}2 \mathrm{D} & =\text { two-dimensional } \\ \mathrm{AM} & =\text { amplitude modulation } \\ \mathrm{EET} & =\text { Energy Efficient Transport } \\ \mathrm{SHL} & =\text { simplified high lift } \\ \mathrm{LE} & =\text { leading edge } \\ \mathrm{VSF} & =\text { vortex shedding frequency }\end{array}$

Subscripts

$\mathrm{m}, \mathrm{M}=$ modulation

peak $=$ maximum measured value

$$
\begin{array}{lll}
\delta_{L E} & =\text { LE flap deflection } \\
\nu & =\text { kinematic viscosity }
\end{array}
$$

$\begin{array}{rll}\text { AFC } & \text { active flow control } \\ \text { BART } & \text { Basic Aerodynamics Research } \\ & \text { Tunnel } \\ \text { LTPT } & \text { Low Turbulence Pressure } \\ & \text { Tunnel } \\ \text { ZMF } & \text { zero-net-mass-flux } \\ \mathrm{TE} & =\text { trailing edge }\end{array}$

$\max =$ maximum value

$\infty \quad=\quad$ freestream conditions

\section{Introduction}

$\mathrm{T}$ HIS paper focuses on the results obtained for a simplified version of the NASA Energy Efficient Transport $12 \%$-thick supercritical airfoil operating in a high-lift configuration through the use of active flow control. This work extends the Reynolds number range of previous results obtained on a smaller version of the mode $]^{1}\left[\begin{array}{l}2 \\ 3\end{array}\right.$ tested in the NASA Langley Basic Aerodynamics Research Tunnel. The current research effort marks the return of this airfoil section to the NASA Langley Low-Turbulence Pressure Tunnel. The airfoil section was extensively tested in this facility as a three-element high-lift configuration. ${ }^{4}$ It is the goal of the current research effort to achieve similar performance with a single-element simplified high-lift system augmented with active flow control. Prior to describing the present experiment and presenting the results some background information is provided.

Experiments at both low ${ }^{\sqrt{5}}$ and high ${ }^{6 / 7}$ Reynolds numbers have demonstrated the ability of periodic excitation to effectively and efficiently control separation. These experiments compared steady momentum transfer methods to periodic excitation and showed the momentum requirements were considerably reduced when using periodic excitation. The efficiency of periodic excitation is attributed to exciting the flow at frequencies that match the natural instabilities of the separating shear layer. A system study by McClean et al $\frac{8}{8}$ indicated that reducing the complexity of high-lift systems, by the application of periodic excitation for controlling separation, would have significant benefits and would represent a high payoff application of this technology. The approach taken was to simplify a conventional three-element high-lift system by replacing the slat and Fowler flap with a simply hinged leading- and trailing-edge flap. The airfoil chosen as a basis for this was the EET airfoil, because of its existing dataset in both cruise and high-lift configurations. ${ }^{4}$ To achieve similar performance to the three-element high lift configuration of the EET, it is expected that larger trailing-edge flap deflections would be required on the simplified EET high-lift system and the application of active flow control near the leading- and trailing-edge flap shoulder would enable the simplified system to generate lift comparable to the three-element high-lift configuration.

Low Reynolds number results of Melton et al $1 \frac{2 \sqrt[3]{3}}{3}$ showed that periodic excitation was effective at controlling leading-edge flap shoulder separation, increasing the maximum lift coefficient, $C_{L, \max }$, by 10 $15 \%$ at low (less than $5^{\circ}$ ) trailing-edge flap deflections. Amplitude modulation of the high frequency resonant based piezoelectric actuators used during the experiments to achieve reduced frequencies comparable to those used in the experiments of Seifert et al .5$] 6.7$ reduced the momentum required by an additional $50 \%$ to $70 \%$, while maintaining the critical peak velocity of the actuator. Multiple excitation locations all controlled by a single actuator were available for controlling separation from the trailing-edge flap shoulder. It was demonstrated that for each slot location there was an optimal trailing-edge flap deflection that was most effective. In the highly curved region downstream of the trailing-edge flap shoulder, small changes 
in excitation slot locations resulted in large changes in the optimal trailing-edge flap deflection angle for lift increment. The momentum requirements for controlling flow separation on the trailing-edge flap were large when compared to other airfoil configuration $\sqrt[5]{[6}$ and to the momentum required for leading-edge flap shoulder control ${ }^{1}$ Additional testing ${ }^{2}$ indicated that coupling excitation from a slot located just upstream of the trailing-edge flap with excitation from a slot on the trailing-edge flap led to an improvement in airfoil performance. Similar to the leading-edge flap shoulder control, the output from high frequency resonant based actuators was modulated to lower frequencies. The flowfield was shown to be sensitive to the relative phase between two actuators when actuators with different resonant frequencies were modulated at the same frequency. For the conditions documented at BART, ${ }^{2}$ the optimal phase relation was found to be $\pm 30^{\circ}$.

The results from the BART version of the EET SHL model1 $2 \frac{3}{3}$ were promising and led to the present study of the model in the LTPT. The organization of this paper is as follows, first results from the current research effort are compared to that of Lin and Dominik ${ }^{4}$ for the cruise configuration. Then, the AFC results are presented when control is applied upstream of the trailing-edge flap. The effects of frequency, amplitude and Reynolds number are presented. Similar results follow for the case where excitation is introduced from the trailing-edge flap of the model. Finally, the excitations from the upstream and downstream actuators are combined and the effect documented.

\section{Wind Tunnel Description}

The current experimental effort was conducted in the NASA Langley Low-Turbulence Pressure Tunnel, which is a single-return closed-throat pressurized wind tunnel ${ }^{[9}$ The tunnel can be pressurized from 1 to 10 atmospheres allowing for independent variation of Mach and Reynolds numbers. The test section is rectangular in shape, $0.914 \mathrm{~m}$ (3 feet) wide, $2.286 \mathrm{~m}$ ( 7.5 feet) high and $2.286 \mathrm{~m}$ (7.5 feet) long. The tunnel has a Mach number range of 0.05 to 0.5 , a Reynolds number range of $1.3 \times 10^{6} / \mathrm{m}\left(0.4 \times 10^{6} / \mathrm{ft}\right)$ to $49.2 \times 10^{6} / \mathrm{m}\left(15 \times 10^{6} / \mathrm{ft}\right)$, and a stagnation pressure range of $101.325 \mathrm{kPa}(14.7 \mathrm{psi})$ to $1013.25 \mathrm{kPa}(147$ psi). LTPT features a large, 17.6 to 1 , contraction ratio and a set of nine anti-turbulence screens resulting in extremely low levels of freestream turbulence intensity, less than $0.05 \%$ for most cases ${ }^{10}$ A schematic of the facility can be seen in Fig. 1(a) and a photograph of the EET-SHL model installed in the LTPT test section can be seen in Fig. 1(b), The tunnel is equipped with a wake rake comprised of twenty five total pressure probes. The wake rake is located approximately 1.34 (reference) airfoil chords, c, downstream of the center of rotation of the tunnel endplates or $0.84 \mathrm{c}$ downstream of the model trailing edge.

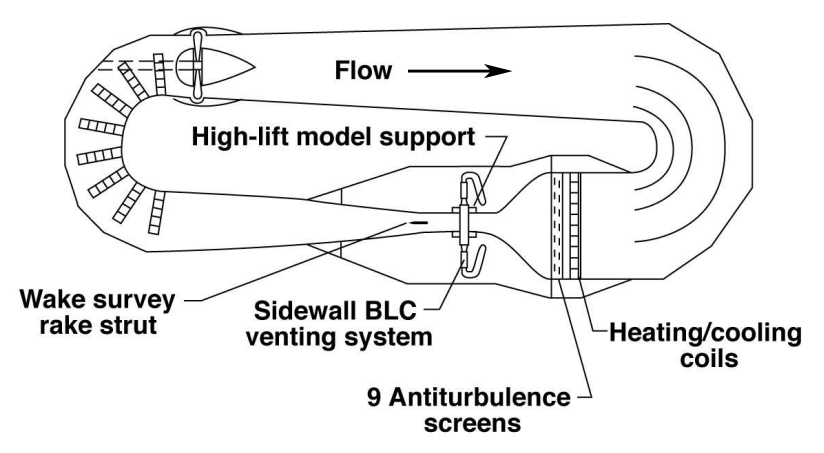

(a) Schematic of the LTPT circuit

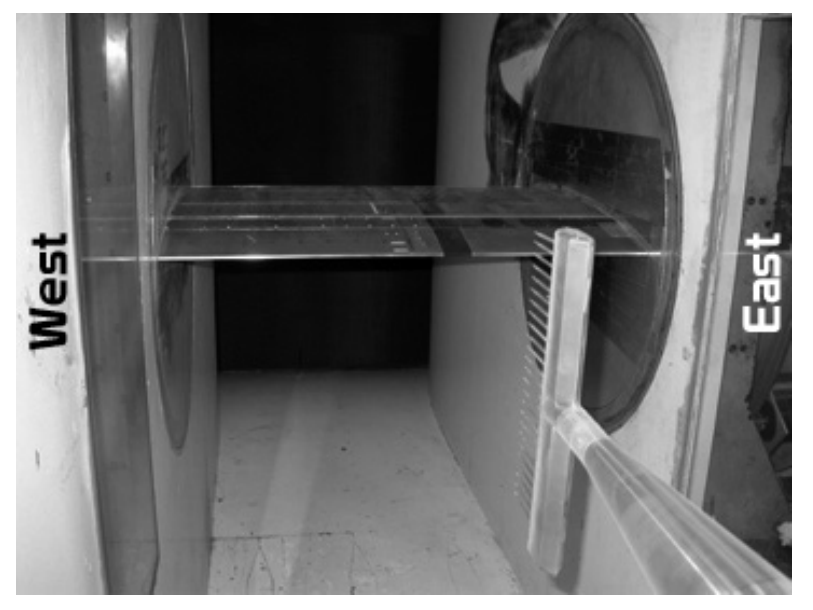

(b) Photograph of the test section with the SHL airfoil model installed, looking upstream.

Figure 1. The NASA Langley Low-Turbulence Pressure Tunnel (LTPT). 


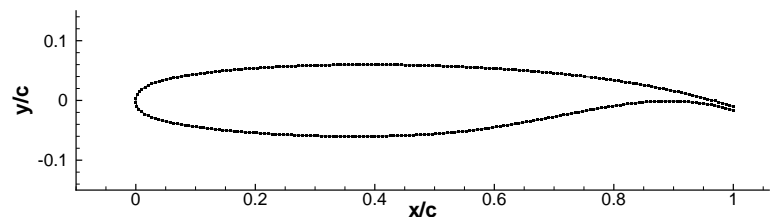

(a) Coordinates of the cruise EET configuration.

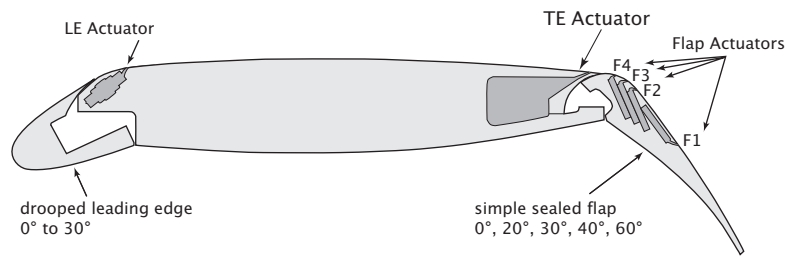

(b) Flaps deflected and actuator locations.

Figure 2. The Simplified High-Lift version of the NASA EET airfoil.

\section{Model Description}

The simplified high-lift version of the NASA EET airfoil utilized here is shown in Fig. 2. The model has a cruise (reference) chord length, c, of $0.762 \mathrm{~m}$ (30.0 inches) and features a $15 \%$ chord leading-edge flap that can be deflected from $0^{\circ}$ to $-30^{\circ}$ and a $25 \%$ chord trailing-edge flap that can be deflected from $0^{\circ}$ to $60^{\circ}$. The flow control results that will be presented are for a leading-edge flap deflection, $\delta_{L E}$, of $-25^{\circ}$, and a trailingedge flap deflection, $\delta_{T E}$, of $30^{\circ}$ at $\mathrm{M}=0.1$. The two-dimensional model completely spans the test section of LTPT and is mounted on two endplates, at the East and West sides (right and left sides, respectively, looking upstream) of the tunnel, which attach the model to its support and an integrated balance. The model is equipped with 270 static pressure taps, where 144 taps are distributed along the centerline of the model and 42 taps on each of two streamwise lines located 6 inches from each endplates. There are three spanwise rows, each with 14 taps, located at $\mathrm{x} / \mathrm{c}=0.10,0.35$, and 0.94 . Additionally, the model is equipped with several dynamic pressure transducers on the model surface.

\section{AFC Actuators}

One motivation for the LTPT test was to increase the options for AFC actuators that could be utilized in the experiment, due to a physically larger model. For the smaller BART model, all actuation was done using internal piezoelectric actuators. Also, on the flap of the BART model, one actuator was used to drive three slots via a common cavity. This significantly limited the flow out of multiple slots and essentially limited the study to a single slot on the trailing-edge flap. The LTPT model has 6 independently controlled actuators for applying excitation at 6 locations - one on the shoulder of the leading edge, one on the shoulder of the trailing-edge flap, and four on the trailing-edge flap itself (see Fig. 2(b)).

The naming convention for the actuators follows that shown in Fig. [2(b)] and in Melton, Yao, and Seifert! ${ }^{3}$ The actuator located at the shoulder of the leading-edge flap is the leading-edge actuator, the actuator located immediately upstream of the trailing-edge flap is the trailing-edge actuator and the actuators on the flap itself are referred by number, Flap \#1 (F1) is the actuator closest to the trailing edge of the model, Flap \#4 (F4) is the slot on the flap closest to the leading edge of the model. The slot heights, $\mathrm{h}$, for all actuators are nominally $0.635-0.762 \mathrm{~mm}(0.025-0.030$ inches). A summary of the type of actuators used and their performance is provided in Table 1 .

\section{A. Leading-Edge Actuator}

The leading edge (LE) actuator assembly is a zero-net-mass-flux (ZMF) actuator, which consists of 4 independent internally-mounted electromagnetic actuation units. The excitation covered the center $64 \%$ span of the model and the AFC slot was located at $\mathrm{x} / \mathrm{c}=0.14$. The electromagnetic actuation units were driven in pairs, each pair on a channel of a stereo audio amplifier supplied with a common signal. Typical signal supplied to the actuators was 30 Volts RMS with a peak velocity of around $30 \mathrm{~m} / \mathrm{s}$ and a frequency range of $80-200 \mathrm{~Hz}$ (Table 1). 


\section{B. Trailing-Edge Actuator}

The excitation from the trailing-edge actuator (TE actuator) assembly, located at $\mathrm{x} / \mathrm{c}=0.73$, was created by four externally mounted ZMF actuation units. The output from the four actuation units were connected to a common plenum inside the model by sealed pipes that went through the model endplates. Physically, there was a pair of actuation units mounted on the outside of the model endplate on the East side and on the West side of the tunnel. Electrically, one actuation unit on each side of the tunnel formed a pair with an actuation unit on the other side and each of these pairs was connected to a channel of a stereo audio amplifier supplied with a common signal. The typical signal supplied to each pair of actuators was 30 Volts RMS, resulting in a peak velocity of about $100 \mathrm{~m} / \mathrm{s}$ and a frequency range of $50-200 \mathrm{~Hz}$ (Table 1).

The spanwise uniformity of the TE actuator excitation was assessed prior to the wind tunnel test. Hotwire measurements acquired at the 3 streamwise pressure tap locations are presented in Fig. 3. During the calibration, data were not acquired below $100 \mathrm{~Hz}$ using 30 Volts RMS, which is the cause of the apparent shift in Fig. 3(c), The external actuator arrangement produces the maximum output at the centerline, with the excitation on the West side of the model being the lowest. The ensemble averaged time histories presented in Fig. 4 from the calibration data indicate that the difference in phase between the centerline output and either the East or West output is about 12.5 degrees at $150 \mathrm{~Hz}$ operating frequency.

The performance of the TE actuator was observed to degrade as the total pressure of the tunnel was increased. To achieve the $R e_{c}=1.7 \times 10^{6}$ and $\mathrm{M}=0.1$ condition, the tunnel was run at atmospheric pressure (14.7 psia). At the $R e_{c}=3.4 \times 10^{6}$ and $\mathrm{M}=0.1$ condition, the tunnel total pressure was raised to 29 psia and at the $\mathrm{M}=0.1$ and $R e_{c}=6.0 \times 10^{6}$ and $9.0 \times 10^{6}$ conditions, the tunnel total pressure was 50 psia and 75 psia, respectively. The output from the TE actuator was characterized by making hot-wire measurements of the exit velocity as a function of drive frequency and amplitude. The RMS of the jet exit velocity, $U_{j}$, as a function of frequency is shown in Fig. 5 . The hot-wire was calibrated at the same elevated pressures using a calibration jet prior to acquiring the characterization data. In addition to the hot-wire, a total pressure probe utilizing a dynamic pressure transducer was positioned at the slot exit at a different spanwise location. For the TE actuator, the hot-wire data proved to be unreliable. The data from the total pressure probe indicates that the output velocity of the actuator, for a fixed drive amplitude, is reduced at elevated pressure. This trend is consistent with other AFC actuator characterizations made at elevated total pressures by the authors in the same facility. At elevated total pressures, the output from the actuator is reduced and frequency at which the peak velocity is observed shifts slightly towards lower frequencies. Since the total pressure probe was not dynamically calibrated, only the data at the lowest sampled frequency is considered reliable to provide an indication of how the performance is effected by the elevated total pressures. Based on measurements made with the total pressure probe at 50 Hertz drive frequency, the performance of the TE actuator at 29 psia was reduced to $75 \%$ of that at atmosphere, reduced to $57 \%$ at 50 psia, and reduced to $44 \%$ at 75 psia. These values are indicated by the single points presented in Fig. 5 .

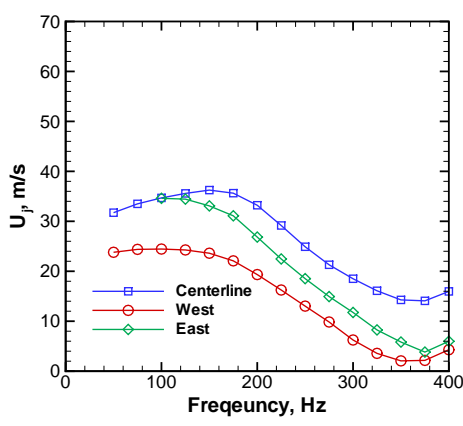

(a) $\mathbf{V}_{\mathbf{R M S}}=10$.

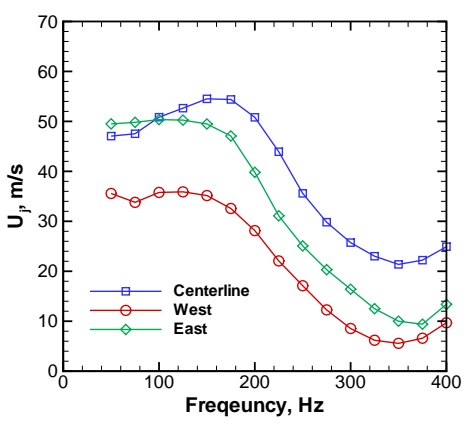

(b) $\mathbf{V}_{\mathbf{R M S}}=20$.

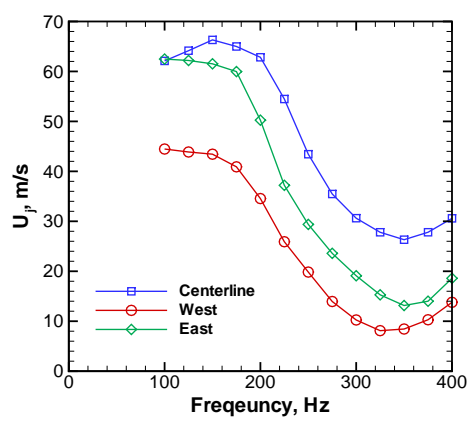

(c) $\mathbf{V}_{\mathrm{RMS}}=30$.

Figure 3. TE actuator calibration at three different spanwise locations. 


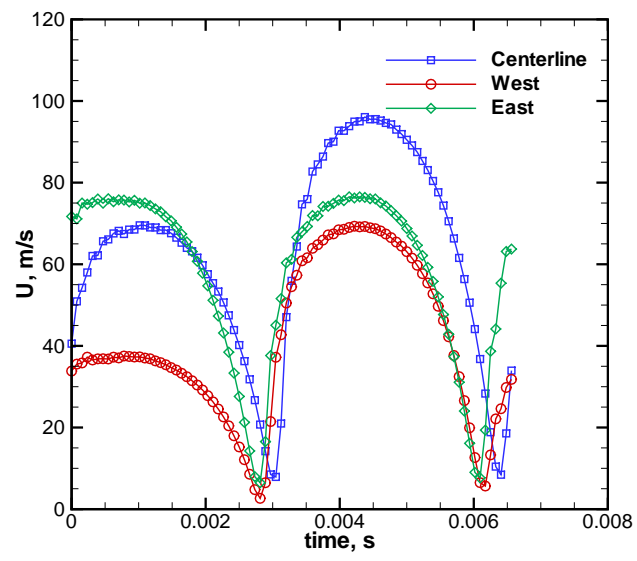

Figure 4. TE actuator ensemble average hot-wire velocity measurements at $\mathrm{f}=150 \mathrm{~Hz}$ and $30 \mathrm{~V}_{\mathrm{RMS}}$ input.

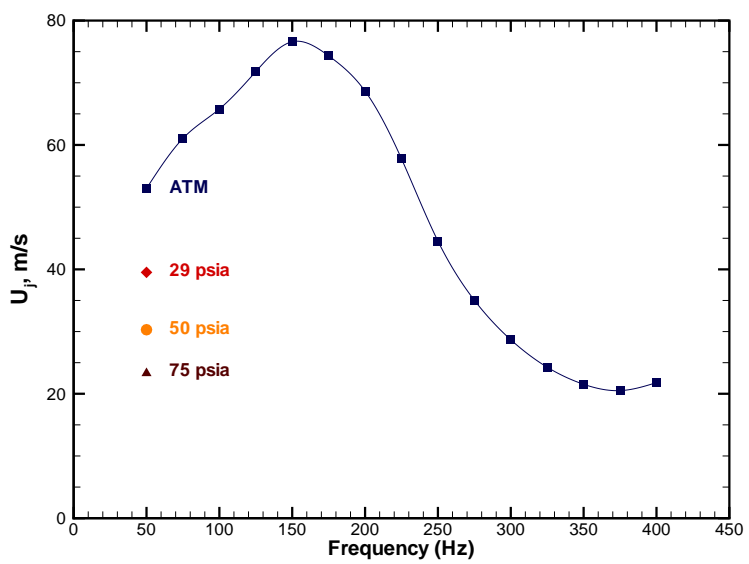

Figure 5. TE actuator frequency sweep at different tunnel total pressures, $30 \mathrm{~V}_{\mathrm{RMS}}$. Velocity measurements at atmosphere are from hot-wire measurements, at elevated pressures total pressure probe.

\section{Trailing-Edge Flap Actuators}

The low Reynolds number SHL results on the BART mode $2[3]$ indicated that the momentum required to control flow separation on the trailing-edge flap of the model was significantly higher than the momentum required for controlling leading-edge flow separation and higher than the momentum required for controlling flow separation on the NACA 0015 at flight Reynolds numbers. The BART data ${ }^{2}$ also indicated that combining multiple excitations would increase control authority. Furthermore, the BART data ${ }^{3}$ indicated that the chordwise spacing of the excitation slots should be closer together around the trailing-edge-flap knee due to the sensitivity to slot location in this highly curved region of the model. Table 1 presents the slot locations that were chosen based on the low Reynolds number database. The trailing-edge flap actuators (TE flap actuators) used were a major upgrade from the ones used in BART and the size of the model enabled four actuator assemblies to be placed in the trailing-edge flap of the model. Each flap actuator assembly consisted of a linear array of 19 piezoelectric actuation units. Each array was independent of the others and was divided in half for the purpose of electronic excitation. The division of the array of 19 actuation units was done in a way that the phase between spanwise adjacent actuation units could be varied. For each of the TE flap slots, half of the piezoelectric actuators were connected to one channel of a stereo audio amplifier and the other half were connected to the other channel. The typical signal supplied to each array of actuators was 100 Volts RMS with a peak velocity of approximately $50 \mathrm{~m} / \mathrm{s}$ at f=1500 $\mathrm{Hz}$ (Table 1).

\begin{tabular}{|l|l|l|l|l|}
\hline Actuator & Type & x/c Location & Frequency Range & U peak,max \\
\hline \hline Leading Edge & Electromagnetic & 0.14 & $80-200 \mathrm{~Hz}$ & $30 \mathrm{~m} / \mathrm{s}$ \\
\hline Trailing Edge & Electromagnetic & 0.73 & $50-200 \mathrm{~Hz}$ & $100 \mathrm{~m} / \mathrm{s}$ \\
\hline Flap \#1, F1 & Piezoelectric & 0.84 & $1500 \mathrm{~Hz} \dagger$ & $50 \mathrm{~m} / \mathrm{s}$ \\
\hline Flap \#2, F2 & Piezoelectric & 0.76 & $1500 \mathrm{~Hz} \dagger$ & $50 \mathrm{~m} / \mathrm{s}$ \\
\hline Flap \#3, F3 & Piezoelectric & 0.74 & $1500 \mathrm{~Hz} \dagger$ & $50 \mathrm{~m} / \mathrm{s}$ \\
\hline Flap \#4, F4 & Piezoelectric & 0.73 & $1500 \mathrm{~Hz} \dagger$ & $50 \mathrm{~m} / \mathrm{s}$ \\
\hline
\end{tabular}

Table 1. Actuator types by location. †Lower frequencies were obtained with the piezoelectric actuator using amplitude modulation. 


\section{Cruise and High-Lift Baseline Airfoil Performance}

The cruise configuration $\left(\delta_{L E}=\delta_{T E}=0^{\circ}\right)$ of the model was tested prior to deflecting the leading- and trailing- edge flaps and applying active flow control as was done with the BART model ${ }^{1}$ The SHL BART dataset of Melton et al ${ }^{1}$ was acquired at Reynolds numbers much lower than the data of Lin. $\underline{4}$ However, a comparison at the same $R e_{c}$ can be made based on data from the current research effort. Figure 6 compares the lift values at $R e_{c}=2.5 \times 10^{6}$ and $R e_{c}=9 \times 10^{6}$. The SHL has a much larger slope change with Reynolds number when compared to the data of Lin. The increase in the stall angle with Reynolds number for the SHL model is due to the sidewall venting utilized for the SHL data. The data of Lin were acquired without sidewall venting and with the sidewall venting holes for boundary-layer control taped. The SHL model was observed to stall sooner (between $15^{\circ}$ and $16^{\circ}$ ) at $R e_{c}=9 \times 10^{6}$ when the endplates were taped and no sidewall venting used. The large Reynolds number sensitivity seen with the SHL EET model is believed to be primarily due to blockage effects that are larger for this $0.762 \mathrm{~m}$ chord model when compared to the 0.55 $\mathrm{m}$ chord model of Lin. When wall corrections ${ }^{11}$ are applied to the data there is a closer agreement between the lift data for the two models (Figure 6).

The high-lift configuration for the model combines a leading-edge flap deflection and a trailing-edge flap deflection $\left(\delta_{L E}=-25^{\circ}, \delta_{T E}=30^{\circ}\right)$. Figure 7 compares the pressure distribution on the BART SHL model to those on the LTPT SHL model. The $C_{p}$ distributions presented, at $\alpha=8^{\circ}$, indicate the presence of a laminar separation bubble on the low Reynolds number BART model at the LE flap shoulder $(\mathrm{x} / \mathrm{c}=0.15)$. The lower surface $C_{p}$ values are almost identical although there are differences near the hinge locations, $\mathrm{x} / \mathrm{c}=0.25$ and $\mathrm{x} / \mathrm{c}=0.75$, for the two datasets. Wind tunnel wall interference is the reason for larger $C_{L}$ generated by the BART model. The solid blockage for the BART model is about $70 \%$ higher than that of the LTPT model. The baseline $C_{p}$ distributions also indicate that the TE flap is completely separated for all cases studied.

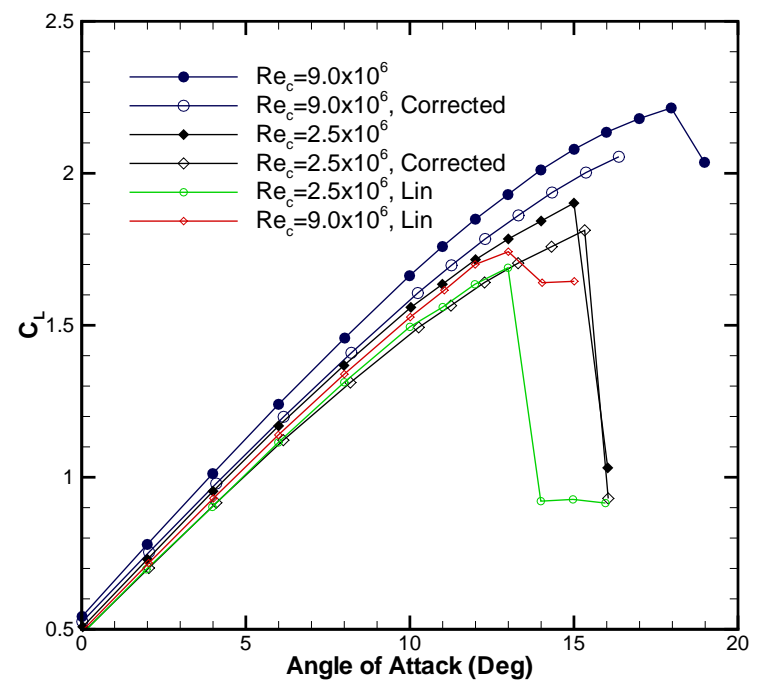

Figure 6. Lift coefficients for the cruise configuration of the EET model.

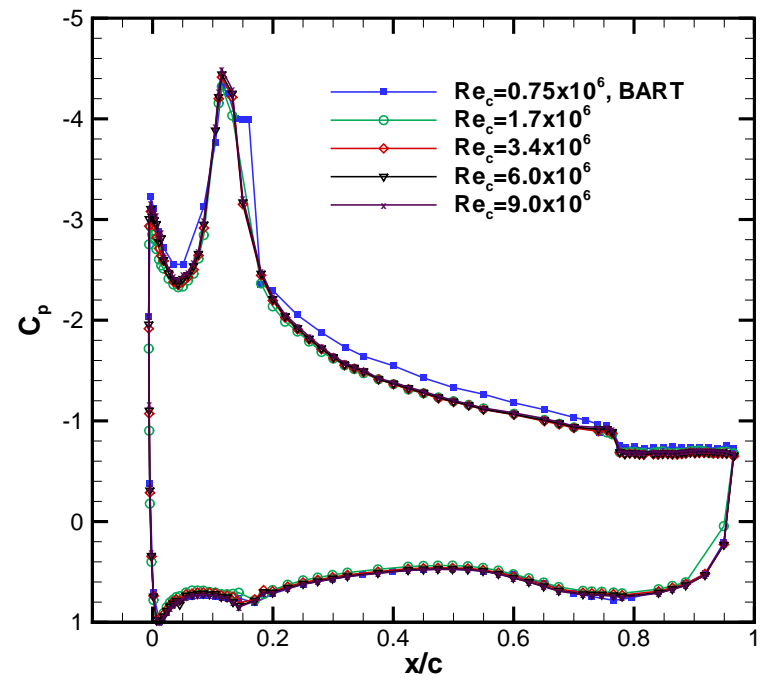

Figure 7. Baseline $\mathrm{C}_{\mathrm{p}}$ distribution comparing BART data to LTPT data. $\mathrm{M}=0.1, \alpha=8^{\circ}$.

\section{TE Actuator Control Results}

\section{A. Effect of TE Actuator Excitation Frequency}

The effects of changing the frequency of the TE actuator on $C_{L}$ are presented in Fig. 8(a) at two angles of attack $\left(\alpha=0^{\circ}\right.$ and $\left.8^{\circ}\right)$. The data, acquired at $R e_{c}=1.7 \times 10^{6}$, indicate that $F^{+}=0.44$ and 0.89 are the best frequencies in the range presented for increasing $C_{L}$. Comparable lift increments are obtained with $F^{+}=0.44$ and $F^{+}=0.89$ at $\alpha=0^{\circ}$. At $\alpha=8^{\circ}, F^{+}=0.89$ is not as effective as $F^{+}=0.44$ at increasing lift. The form drag data shown in Fig. 8(b) indicate that $C_{d p}$ is increased at $\alpha=0^{\circ}$ with $F^{+}=0.89$ and at $\alpha=8^{\circ}$ with $F^{+}=0.44$. The sensitivity to $F^{+}$at these two angles of attack provides the basis for the $F^{+}$ 


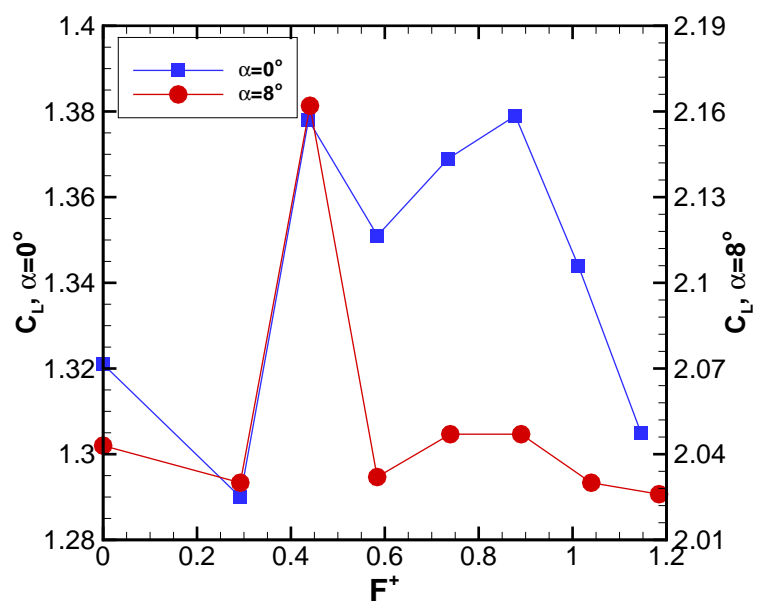

(a) $\mathbf{C}_{\mathbf{L}}$

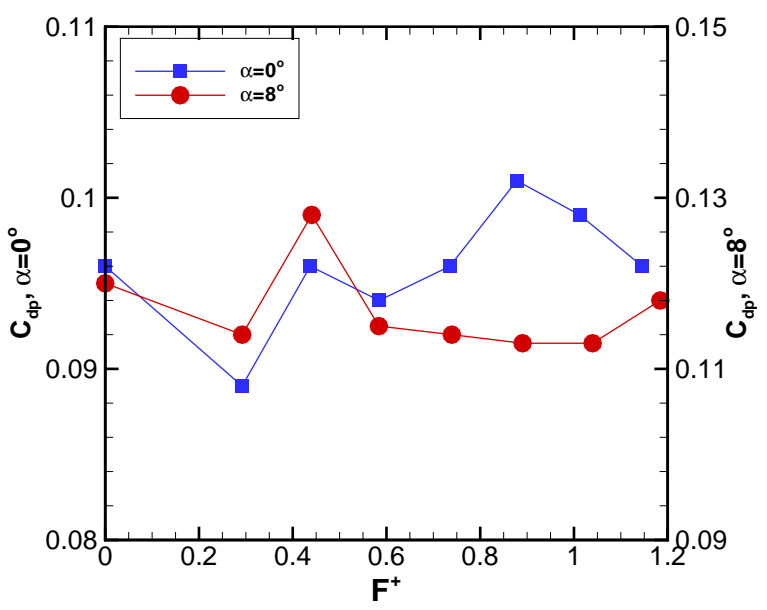

(b) $\mathbf{C}_{\mathbf{d p}}$

Figure 8. TE actuator frequency scan. $\mathbf{R e}_{\mathbf{c}}=1.7 \times 10^{6} ; \delta_{\mathrm{LE}}=-25^{\circ} ; \delta_{\mathrm{TE}}=30^{\circ} ; \mathrm{M}=\mathbf{0 . 1}$.

values used throughout the current research effort. These results are consistent with the results of Melton, Yao, and Seifert ${ }^{2}$ when using excitation from this location on the model.

\section{B. Effect of TE Actuator Excitation Amplitude}

In Fig. 9 the effect of changing the excitation amplitude for the $R e_{c}=1.7 \times 10^{6}$ case is presented for two frequencies; $F^{+}=0.44$ and $F^{+}=0.89$. The results indicate that the $F^{+}=0.44$ case is more effective at increasing the total aerodynamic force, the lift increases above the baseline as does the drag. For the $F^{+}=0.89$ case, both the $\Delta C_{L}$ and the $\Delta C_{d p}$ are initially negative. The $\Delta C_{L}$ reaches a minimum early on and then begins to increase, while the $\Delta C_{d p}$ continues to decrease, which is an indication of less flow separation on the trailing-edge flap. For the $F^{+}=0.89$ excitation case, $\Delta C_{d p}$ begins to increase when $C_{\mu}$ exceeds $0.36 \%$, at which point $\Delta C_{L}$ becomes positive, although the $C_{d p}$ levels continue to remain less than the baseline case $\left(C_{\mu}=0 \%\right)$. The $C_{p}$ distributions at $\alpha=8^{\circ}$ corresponding to $C_{\mu}=0.36 \%$ for the two frequencies are presented in Fig. 10. The data indicate that the $C_{\mu}$ values used are not sufficient to completely eliminate flow separation on the trailing-edge flap. The data also indicate that the two frequencies have different effects on the flowfield. On the trailing-edge flap the higher $F^{+}$excitation increases the model trailing-edge pressures while the $F^{+}=0.44$ case decrease the pressures on the trailing-edge flap. Additionally, the $F^{+}=0.44$ case increases the upper surface suction, thus increasing the overall circulation for the airfoil. In contrast, the $F^{+}=0.89$ case locally improves the $\mathrm{TE}$ pressure recovery indicating a degree of separation control on the flap.

\section{Effect of Reynolds Number}

The data presented in Fig. 11 indicate the effect of varying the excitation amplitude $\left(F^{+}=0.89\right)$ of the actuator at $R e_{c}=1.7 \times 10^{6}$ and $R e_{c}=3.4 \times 10^{6}$. The excitation has the same effect on the flowfield for these two Reynolds numbers. At higher Reynolds numbers, $R e_{c}=6 \times 10^{6}$ and $R e_{c}=9 \times 10^{6}$, this was no longer the case. The differences at higher $R e_{c}$ are believed to be due to actuator output decreasing as tunnel total pressure increases. The actuator was characterized by making velocity measurements via a hot-wire anemometer. These characterizations were performed at total pressures of $50 \mathrm{psi}$ and $75 \mathrm{psi}$, corresponding to $R e_{c}=6 \times 10^{6}$ and $R e_{c}=9 \times 10^{6}$ at $\mathrm{M}=0.1$, and indicated a decrease in actuator output with increasing total pressure (Fig. 5).

The pressure distributions acquired at $R e_{c}=1.7 \times 10^{6}$ (Fig. 12 and $R e_{c}=3.4 \times 10^{6}$ (Fig. 13 provide a possible explanation for the results obtained at $R e_{c}=3.4 \times 10^{6}$ that seem to contradict the hot-wire calibration data. Note, identical dimensional frequencies are used for the two Reynolds numbers of Figs. 12 


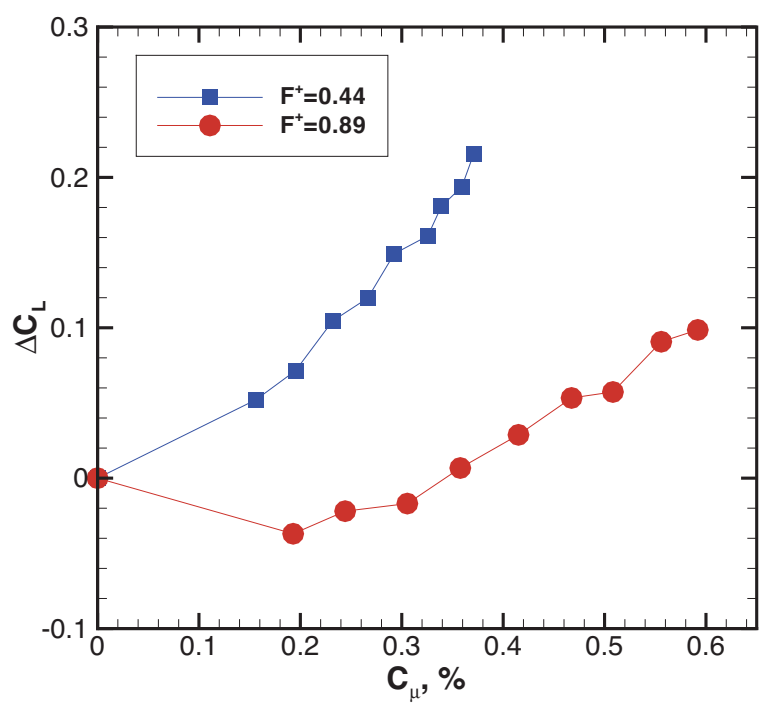

(a) $\Delta \mathbf{C}_{\mathbf{L}}$

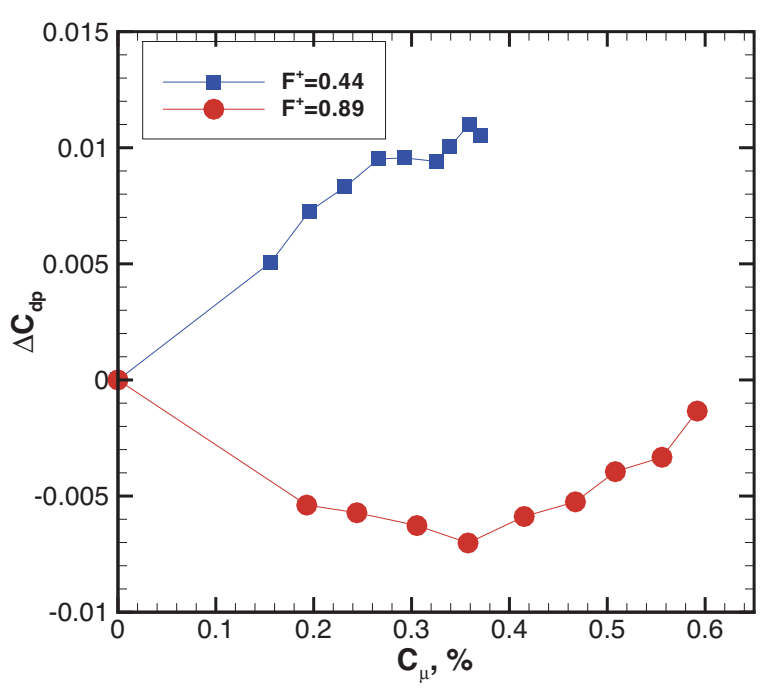

(b) $\Delta \mathbf{C}_{\mathbf{d p}}$

Figure 9. 9 Effect of $\mathrm{TE}$ actuator excitation amplitude on $\Delta \mathrm{C}_{\mathrm{L}}$ and $\Delta \mathrm{C}_{\mathrm{dp}}$. $\operatorname{Re}_{\mathbf{c}}=1.7 \times 10^{6} ; \delta_{\mathbf{L E}}=-25^{\circ} ; \delta_{\mathrm{TE}}=30^{\circ} ; \mathbf{M}=0.1, \alpha=8^{\circ}$.

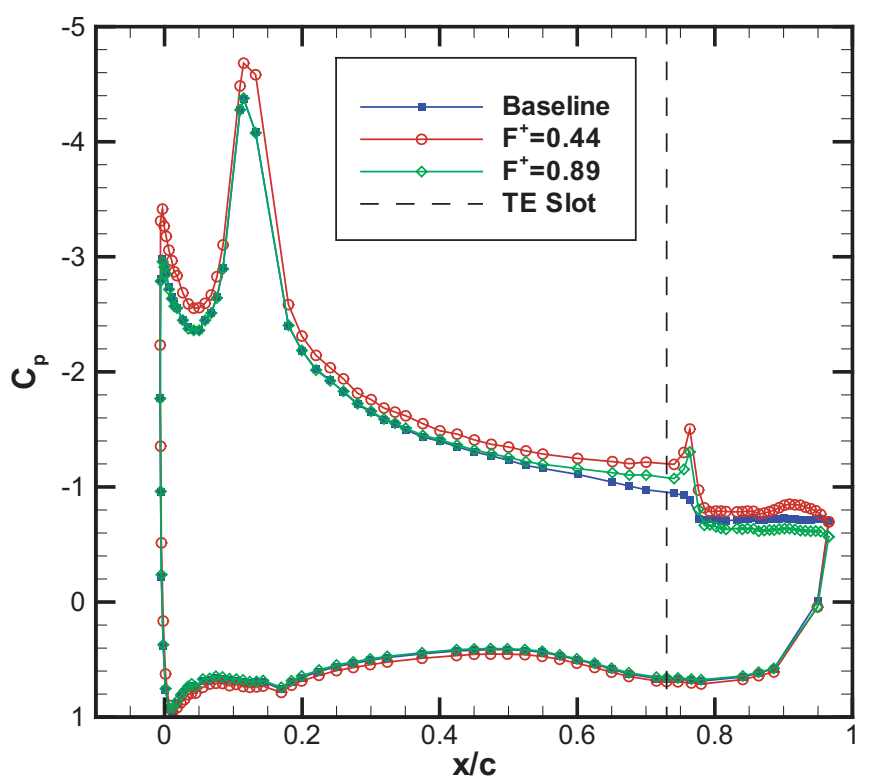

Figure 10. $\mathrm{C}_{\mathrm{p}}$ distribution with $\mathrm{TE}$ actuator control comparing $\mathrm{F}^{+}=0.44$ and $\mathrm{F}^{+}=0.89 . \quad \mathrm{Re}_{\mathrm{c}}=1.7 \times 10^{6} ;$ $\mathrm{M}=0.1 ; \alpha=8^{\circ}$. 


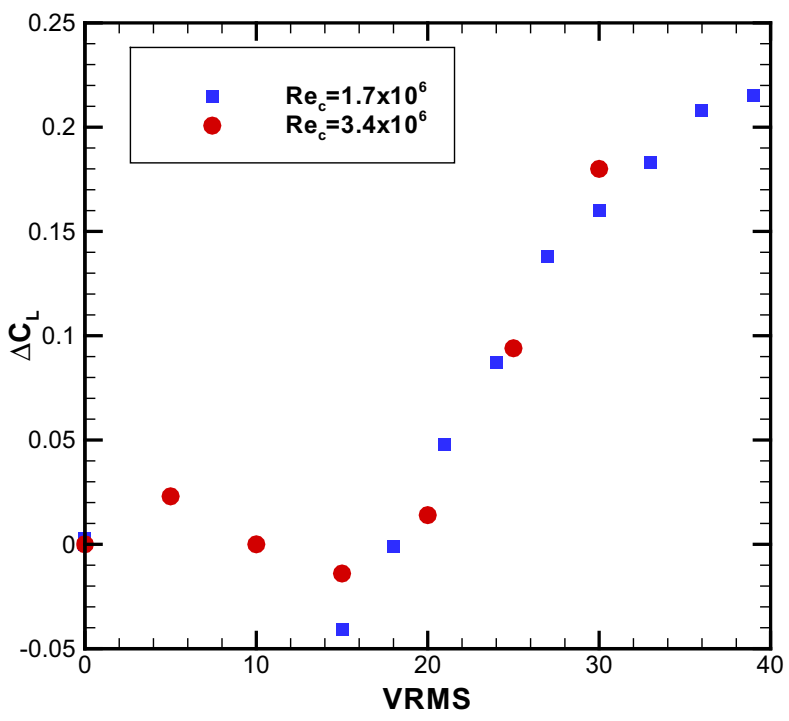

Figure 11. $\Delta \mathrm{C}_{\mathrm{L}}$ vs. VRMS for TE actuator comparing two Reynolds numbers. $\alpha=0^{\circ} ; \mathrm{F}^{+}=0.89$.

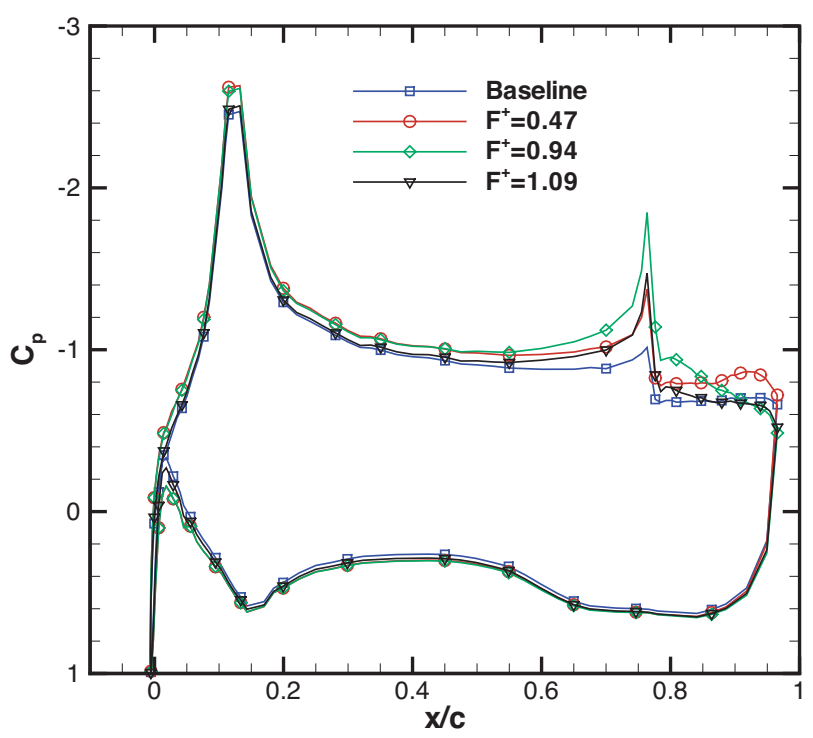

Figure 13. $C_{p}$ distribution for TE actuator excitation. $\operatorname{Re}_{\mathbf{c}}=3.4 \times 10^{6} ; \alpha=0^{\circ}$. Only every fourth data point marked with symbols.

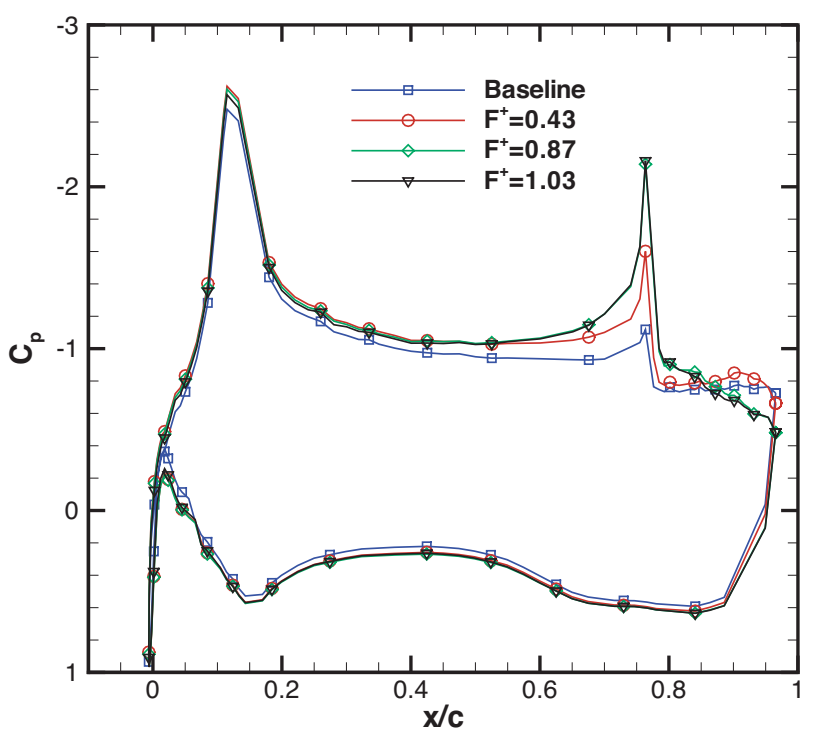

Figure 12. $C_{p}$ distribution for TE actuator excitation. $\operatorname{Re}_{\mathbf{c}}=1.7 \times 10^{6} ; \alpha=0^{\circ}$. Only every fourth data point marked with symbols.

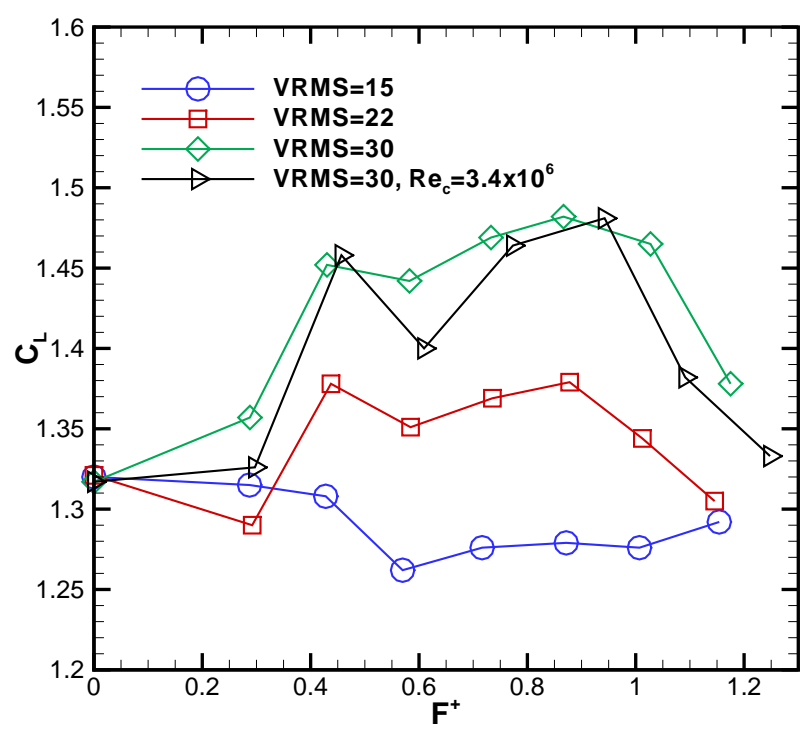

Figure 14. Frequency scan with varied amplitude for TE actuator. $\alpha=0^{\circ} ; \mathbf{R e}_{\mathbf{c}}=1.7 \times 10^{6}$. 
and 13 . The differences in $F^{+}$values are due to different freestream velocities required to generate $\mathrm{M}=0.1$. The data indicate exciting the flow at the natural vortex shedding frequency (VSF), determined by examining the surface pressure spectra, and $2 \times$ (natural VSF), $F^{+}=0.43$ and $F^{+}=0.87$, respectively is more effective for $R e_{c}=3.4 \times 10^{6}$ than at $R e_{c}=1.7 \times 10^{6}(F+=0.43$ and $F+=0.87)$. This provides a possible explanation for the amplitude data of Fig. 11 being comparable at the two Reynolds numbers even though the actuator output is slightly lower at the higher Reynolds number. To further support this theory, the data of Fig. 14 shows the amplitude effect on $C_{L}$ vs. $F^{+}$and also compares the maximum ouput at $R e_{c}=3.4 \times 10^{6}$ with $R e_{c}=1.7 \times 10^{6}$, which indicates that at other frequencies the data appears to be at a lower amplitude. With the exception of $F^{+}=0.43$ and 0.87 , the lower amplitude data of Fig. 14 results in less $\Delta C_{L}$ due to excitation.

\section{Effect of Angle of Attack}

Figure 15 presents lift vs. angle of attack data for the airfoil with control applied at different $F^{+}$values using the TE actuator at $R e_{c}=1.7 \times 10^{6}$. The voltage input to the actuator remains constant. The data indicate that $F^{+}=0.44$ is the most effective frequency for increasing lift over the entire lift curve.

A dynamic pressure transducer located near the TE flap shoulder $(\mathrm{x} / \mathrm{c}=0.77)$ was used to determine the natural vortex shedding frequency of the airfoil, as shown schematically in Fig. 16. Analysis of the power spectra of that pressure signal is presented in Fig. 17 and has been scaled by the tunnel dynamic pressure. Figure 17(b) and 17(c) indicate that with TE actuator control there is more energy in the spectra at higher frequencies and the excitation frequency and its higher harmonics dominate. The baseline data, presented in Fig. 17(a), indicate that the natural vortex shedding frequency of the airfoil is $\mathrm{f}=75 \mathrm{~Hz}\left(F^{+}=0.44\right)$ for $\alpha=0^{\circ}$ but at $\alpha=14^{\circ}$ it has shifted to $\mathrm{f}=56 \mathrm{~Hz}\left(F^{+}=0.33\right)$. This finding coincides with the lift polar of Fig. 15 that indicates that while $F^{+}=0.44$ is effective at increasing lift at low angles of attack, it is not as effective at $\alpha=14^{\circ}$. Additional data acquired at $R e_{c}=3.4 \times 10^{6}$ and presented in Fig. 18 indicate that at $\alpha=14^{\circ}$ a TE Actuator excitation of $F^{+}=0.33$ is more effective at increasing lift than the $F^{+}=0.44$ excitation. The shift in the vortex shedding frequency from $75 \mathrm{~Hz}$ at $\alpha=0^{\circ}$ to $56 \mathrm{~Hz}$ at $\alpha=14^{\circ}$ suggests a scaling with the vertical span of the wake that is supported by the wake data that was acquired.

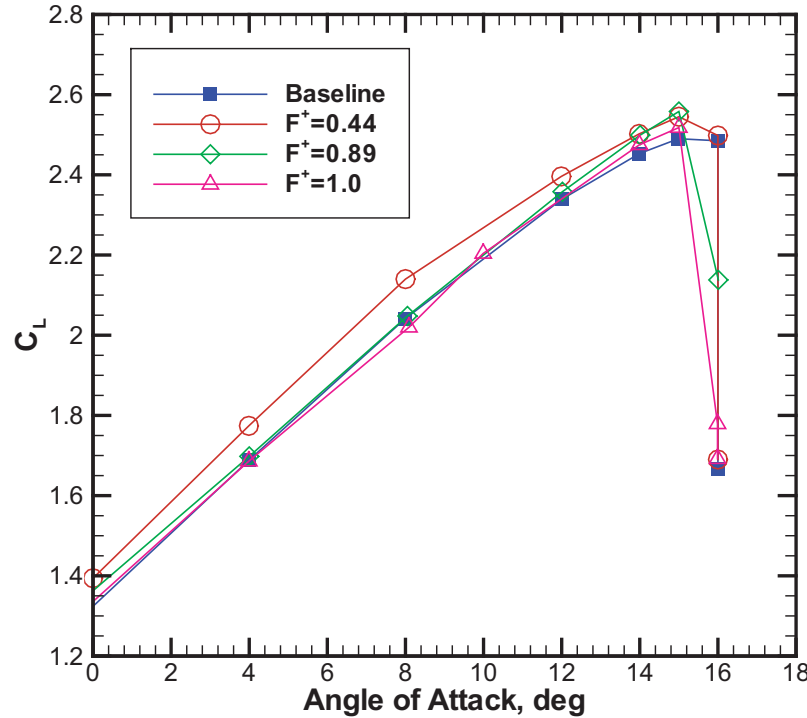

Figure 15. $\mathbf{C}_{\mathbf{L}}$ vs $\alpha$ with $\mathrm{TE}$ actuator control at various $\mathrm{F}^{+}$settings. $\mathrm{Re}_{\mathrm{c}}=1.7 \times 10^{6} ; \mathrm{M}=0.1 ; \mathrm{C}_{\mu} \approx 0.4 \%$

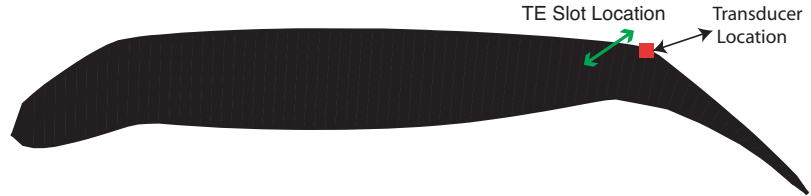

Figure 16. Location of transducer used to determine natural vortex shedding frequency.

\section{TE Flap Actuator Control Results}

The actuators used on the TE flap for controlling separation were flap actuator \#2 (F2) and flap actuator \#3 (F3). Figure 19 presents the $C_{p}$ distributions for the baseline flowfield and the flowfields with control applied using F2 and F3 individually and in combination. The actuators are operated at their resonant 


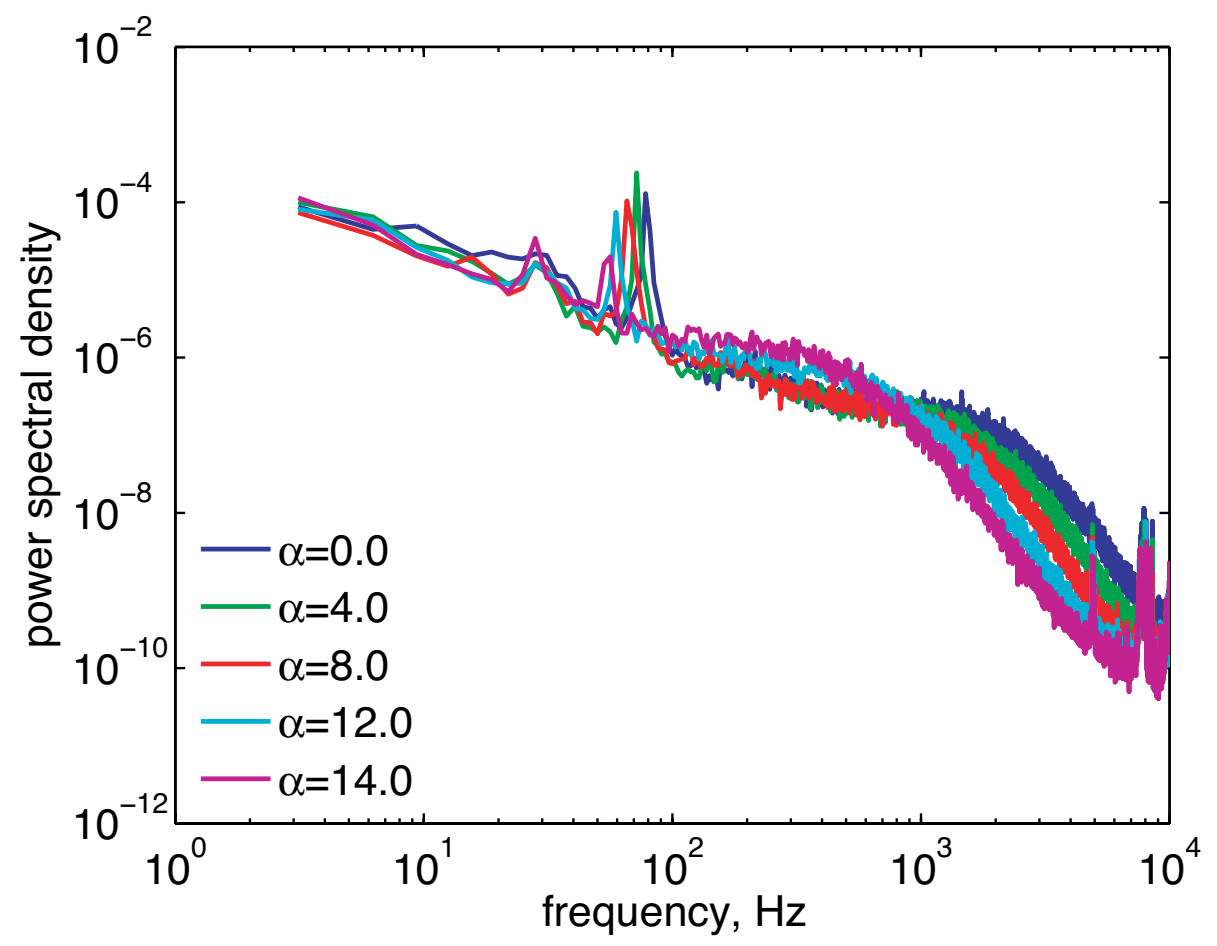

(a) Baseline power spectral density.

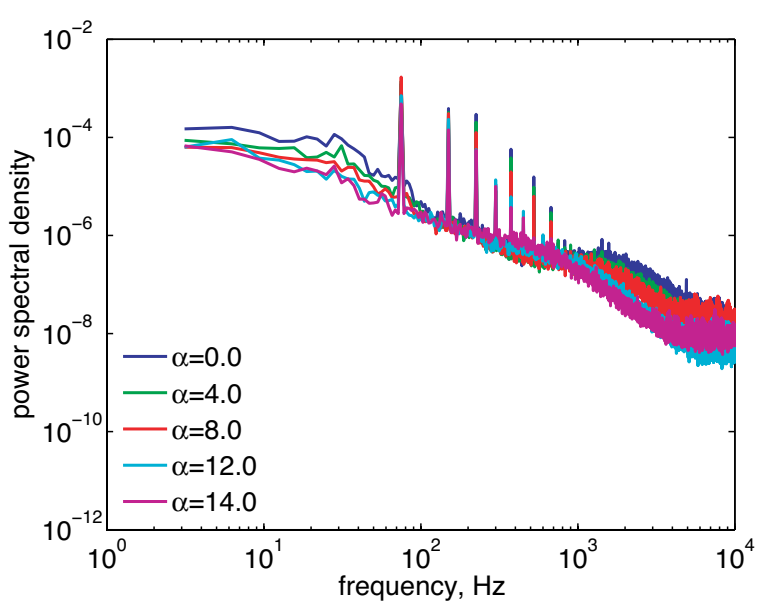

(b) $\mathbf{f}=75 \mathrm{~Hz}$ power spectral density.

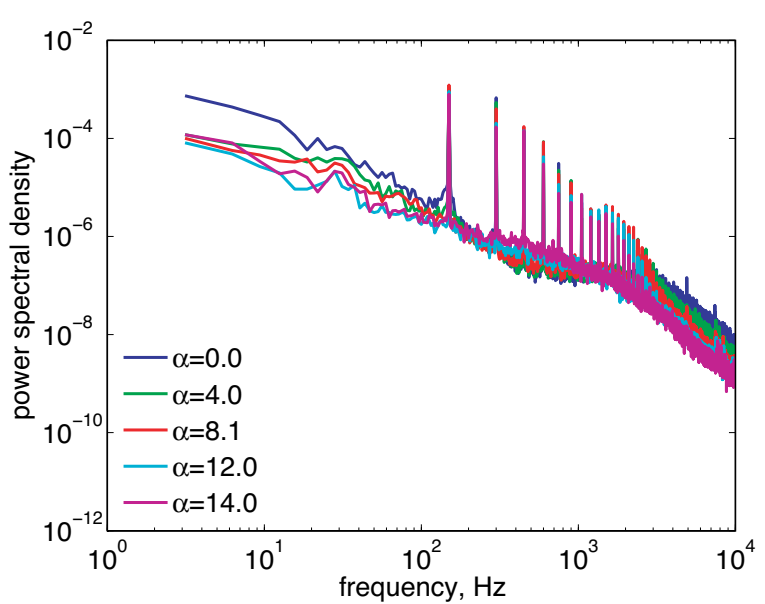

(c) $\mathbf{f}=150 \mathrm{~Hz}$ power spectral density.

Figure 17. Power spectral density with TE actuator control at different frequencies, $\mathrm{C}_{\mu} \approx 0.4 \%$. 


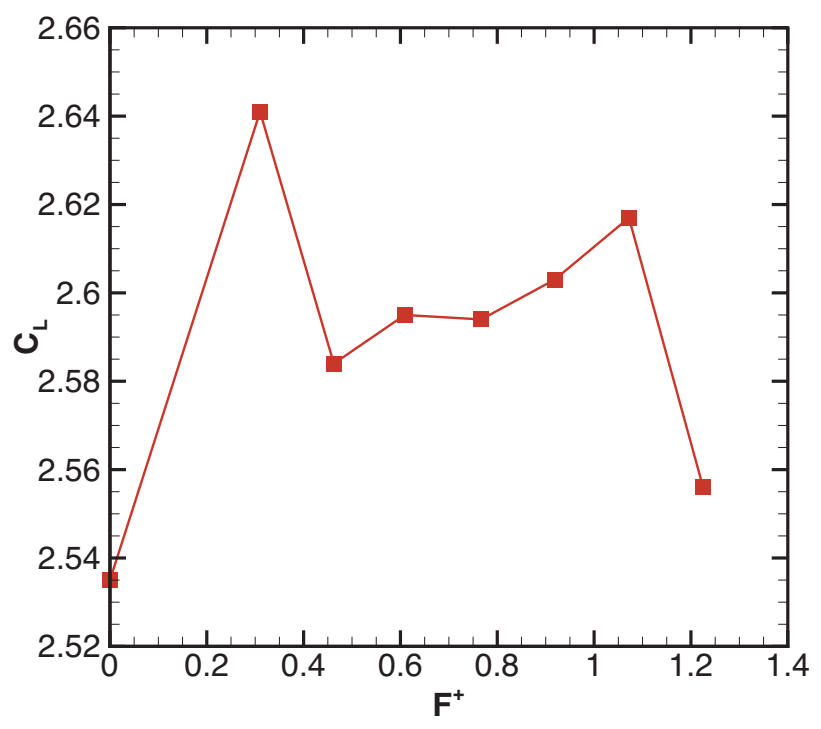

Figure 18. TE actuator $\mathrm{C}_{\mathrm{L}}$ vs. frequency. $\alpha=14^{\circ}$; $\operatorname{Re}_{\mathrm{c}}=3.4 \times 10^{6}$.

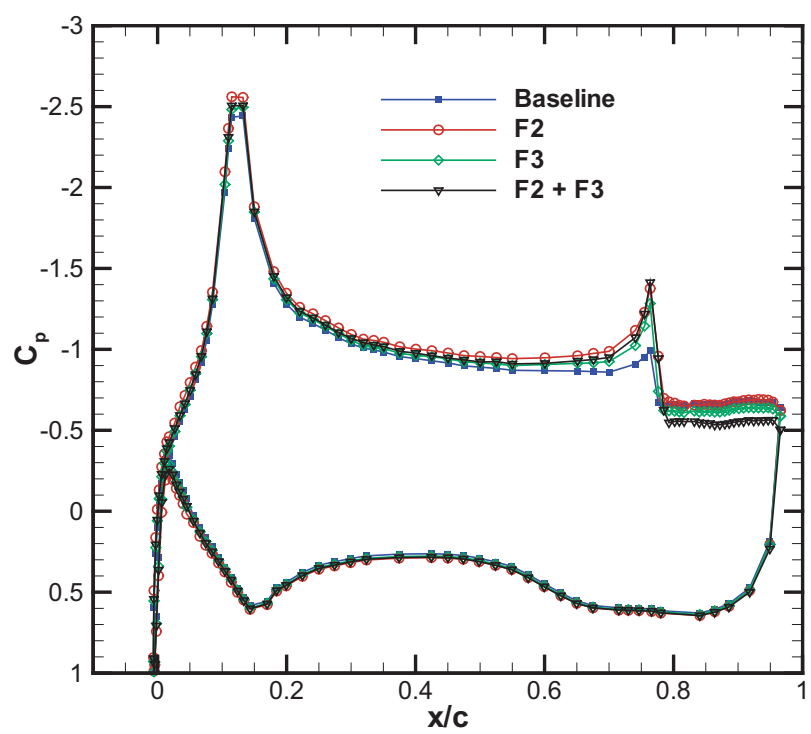

Figure 19. $\mathbf{C}_{\mathbf{p}}$ distribution varying TE flap excitation location. $\operatorname{Re}_{\mathbf{c}}=3.4 \times 10^{6} ; \mathrm{M}=0.1 ; \alpha=0^{\circ} ; \mathrm{C}_{\mu}=0.12 \%$. Actuators were operated at resonance.

frequencies, $\mathrm{f}=1500 \mathrm{~Hz}\left(F_{F 2}^{+}=8.1\right.$ and $\left.F_{F 3}^{+}=8.7\right)$, with maximum actuator output producing $C_{\mu} \approx 0.12 \%$ for each actuator. The data indicate that the two actuators, when operated together, are more effective at controlling separation than operating either actuator individually. For the amplitude levels used during the experiment, the flap actuators operated at resonance are more effective at reducing $C_{d p}$ than increasing $C_{L}$. The lift to drag ratio increases from 14.6 for the baseline flowfield to 18.75 for the (F2 + F3) controlled flowfield. For most of the data to be presented with the TE flap actuator, the two actuators are operated in phase and the high frequency resonant based actuators are amplitude modulated to generate $F^{+}$values of 1 or less.

\section{A. Effect of TE Flap Excitation Frequency}

Figure 20 presents the effect of the modulation frequency of F2 and F3 on $C_{L}$ and $C_{d p}$ for each actuator operating alone. The data indicate that most effective reduced frequencies for increasing lift at $\alpha=0^{\circ}$ are $F_{M}^{+}=0.4$ and $F_{M}^{+}=0.8$, the same reduced frequencies that are the most effective when using the TE actuator. When the angle of attack is increased to $8^{\circ}$ the $F_{M}^{+}=0.4$ excitation from the TE flap actuators (Fig. 21) is still the most effective frequency for increasing lift but $F_{M}^{+}=0.8$ is not effective.

\section{B. Effect of TE Flap Excitation Amplitude}

The effects of varying the actuator amplitude are presented in Figure 22. The data, acquired at $R e_{c}=$ $3.4 \times 10^{6}$, indicate that the largest lift increment is obtained when using $\mathrm{F} 2$ with $F^{+}=7.8$. By modulating the excitation from $\mathrm{F} 2, F_{M}^{+}=0.80$, the momentum required to obtain lift increments above 0.02 is reduced. The lift increment generated when using $\mathrm{F} 3$ with $F^{+}=8.5$ converges to 0.04 . When the excitation from F3 is modulated, $F_{M}^{+}=0.86$, the lift increment is increased by up to 0.03 over that produced by the actuator operating at resonance. Data acquired at $R e_{c}=1.7 \times 10^{6}$ indicate that there is no measurable improvement in $C_{L}$ when modulating F2 and F3 at $F_{M}^{+}=0.80$ and $F_{M}^{+}=0.86$, respectively. The surface pressure spectra on the TE of the model indicate that the excitation frequency locks with the vortex shedding frequency more effectively at $R e_{c}=3.4 \times 10^{6}$ thereby increasing $C_{L}$.

\section{Effect of Reynolds Number}

TE Flap actuators, F2 and F3, were operated at their resonant frequencies $\left(F_{F 2}^{+}=7.8\right.$ and $\left.F_{F 3}^{+}=8.5\right)$ at $\mathrm{M}=0.1$ and the Reynolds number varied from $R e_{c}=1.7 \times 10^{6}$ to $R e_{c}=9.0 \times 10^{6}$. The $C_{p}$ distributions corresponding to these conditions are presented in Fig. 23 . The data of Figure 23 were acquired with the 


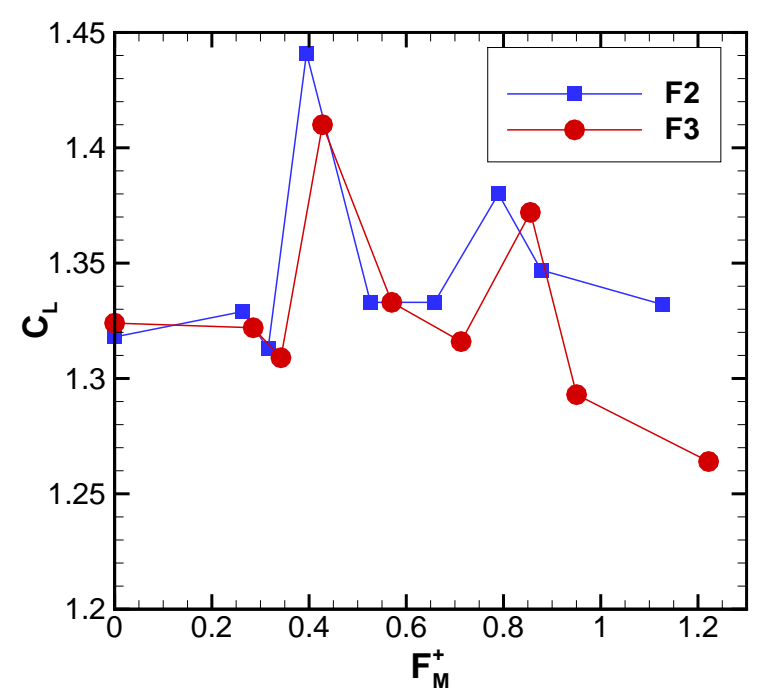

(a) $\mathbf{C}_{\mathbf{L}}$.

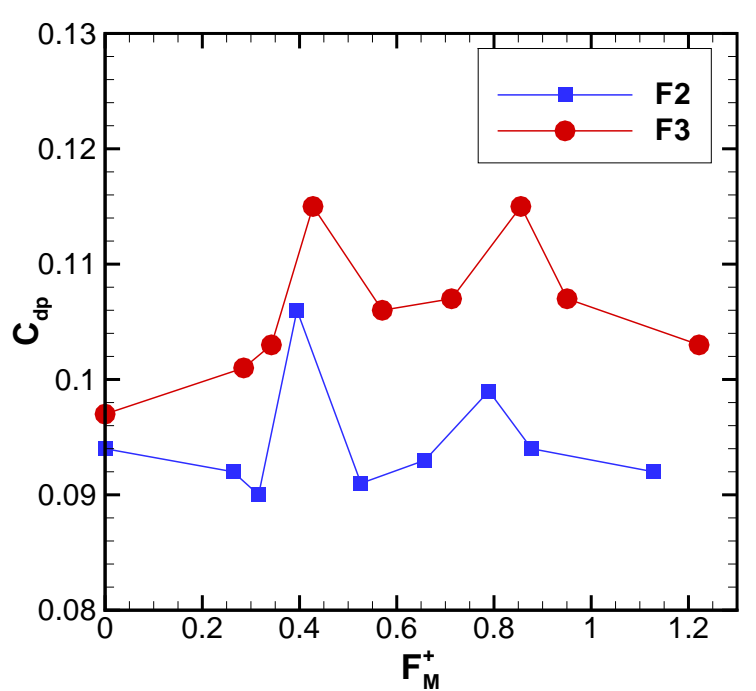

(b) $\mathbf{C}_{\mathbf{d p}}$.

Figure 20. Lift and drag versus frequency with TE flap actuators active. $\alpha=0^{\circ} ; \operatorname{Re}_{\mathrm{c}}=3.4 \times 10^{6}$.

actuators operating at a fixed frequency of $1500 \mathrm{~Hz}$. The data, acquired at $\alpha=8^{\circ}$, indicate that as $R e_{c}$ is increased the ability of the TE flap actuators to control separation is reduced. The flap actuator calibration data using a hot-wire was performed at tunnel total pressures corresponding to the $R e_{c}$ presented. The data indicated the effect of pressure on the piezoelectric actuators is to reduce the resonant frequency of the actuators and to reduce $U_{\text {peak }}$. For example between $R e_{c}=1.7 \times 10^{6}$ and and $R e_{c}=3.4 x 10^{6}$ there is a $15 \%$ reduction in $U_{\text {peak }}$ at $\mathrm{f}=1500 \mathrm{~Hz}$.

\section{TE and Flap Excitations Combined}

Figures 24 and 25 present the $C_{p}$ and wake distributions of the airfoil when control is applied from the TE flap alone or in combination with excitation from the TE flap actuator. Exciting the flow near the vortex shedding frequency with F2 and F3 as presented in Fig. 24 increases the circulation more effectively than exciting the flow at a forcing frequency that is twice the vortex shedding frequency of the airfoil as shown in Fig. 25. The wake measurement presented in Figs. 24 and 25 indicate that the $\mathrm{f}=150 \mathrm{~Hz}$ excitation reduces drag more effectively than the $\mathrm{f}=75 \mathrm{~Hz}$ excitation. At both frequencies presented, combining the TE excitation with excitation from F2 and F3 increases lift and reduces drag more effectively than using the TE flap actuators alone to control separation. When the TE actuator and TE flap actuator excitations are combined, the relative phase between the upstream (TE actuator) and downstream (F2 and F3 actuators) is zero.

Figure 26 presents the lift and drag data obtained when varying the phase between the TE actuator and the TE flap actuators. The TE flap actuators, F2 and F3, are operated in phase and amplitude modulated at $f_{m}=150 \mathrm{~Hz}$ producing $F_{M}^{+}=0.80$ and $F_{M}^{+}=0.86$ for $\mathrm{F} 2$ and $\mathrm{F} 3$, respectively. The TE actuator was operated at $\mathrm{f}=150 \mathrm{~Hz}, F^{+}=0.89$, and the relative phase between the TE and TE flap actuators is varied. The sensitivity to phase between the actuators upstream and downstream of the flap knee are similar to the results of Melton et al $!^{[2}$ The optimal phase for increasing the lift increment of $300^{\circ}$ to $360^{\circ}$ is shifted by $30^{\circ}$ when compared to results of Melton et al ${ }^{2}$ who examined the phase sensitivity of two AM actuators with comparable outputs. Figure 27 presents $C_{p}$ distributions corresponding to a relative phase of zero degrees comparing the effects of combining excitation around the TE flap knee. The data indicate that most of the $C_{L}$ increment is due to the TE actuator. The TE flap actuators alone have more of an upstream effect around the TE flap knee for $R e_{c}=3.4 \times 10^{6}$ and $\mathrm{M}=0.1$. The combination of the excitations upstream and downstream of the TE flap knee provide a synergistic improvement in performance that is larger than the operation of the actuators individually. 


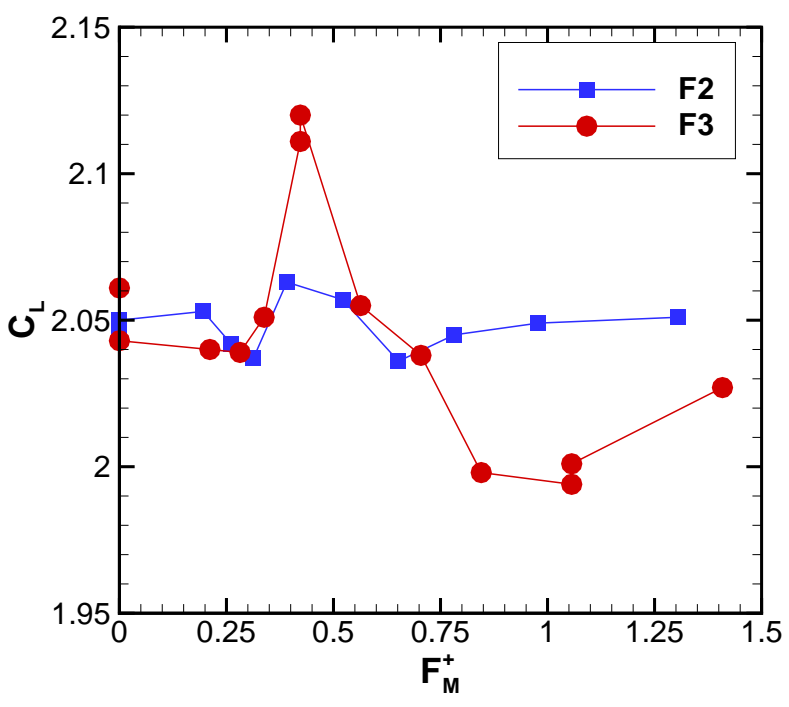

(a) $\mathbf{C}_{\mathbf{L}}$

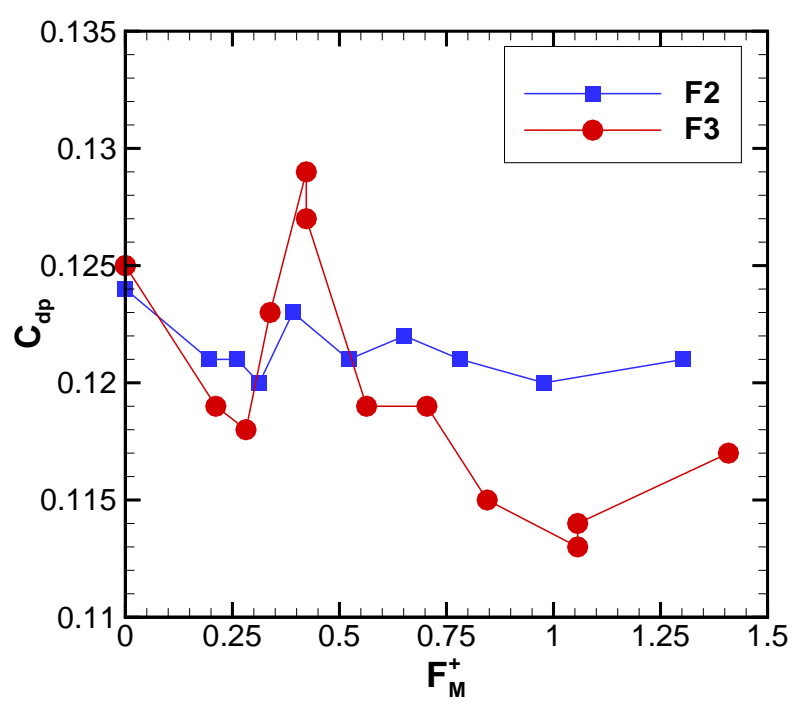

(b) $\mathbf{C}_{\mathbf{d p}}$.

Figure 21. Lift and drag versus frequency with TE flap actuators active. $\alpha=8^{\circ} ; \operatorname{Re}_{c}=1.7 \times 10^{6}$.

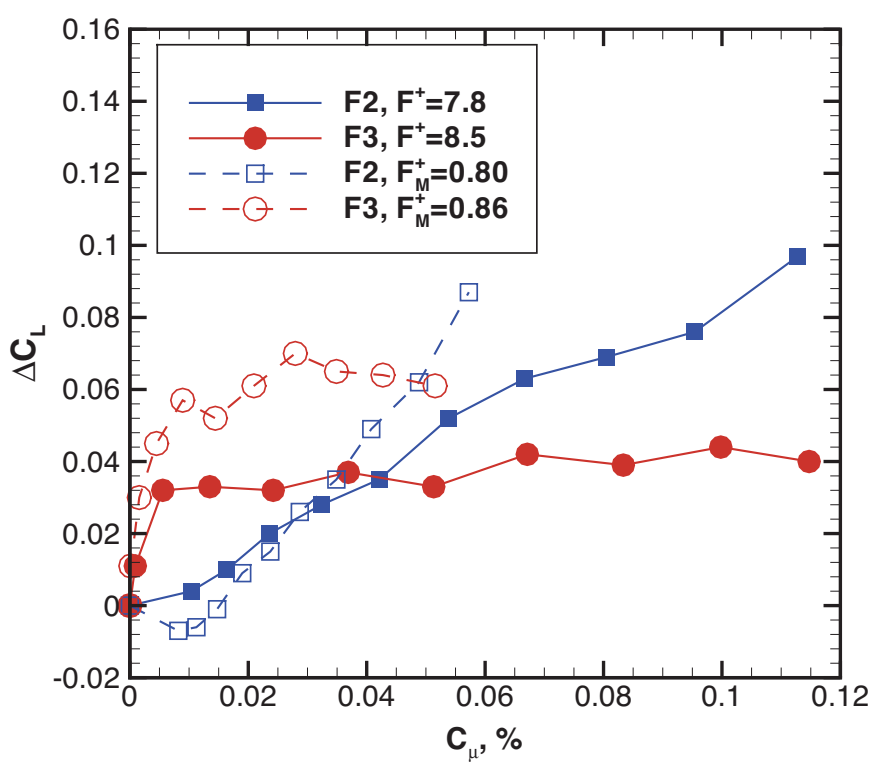

Figure 22. Effect of TE flap actuators, F2 and F3, excitation amplitudes. $\operatorname{Re}_{\mathrm{c}}=3.4 \times 10^{6} ; \mathrm{M}=0.1 ; \alpha=0^{\circ}$. 


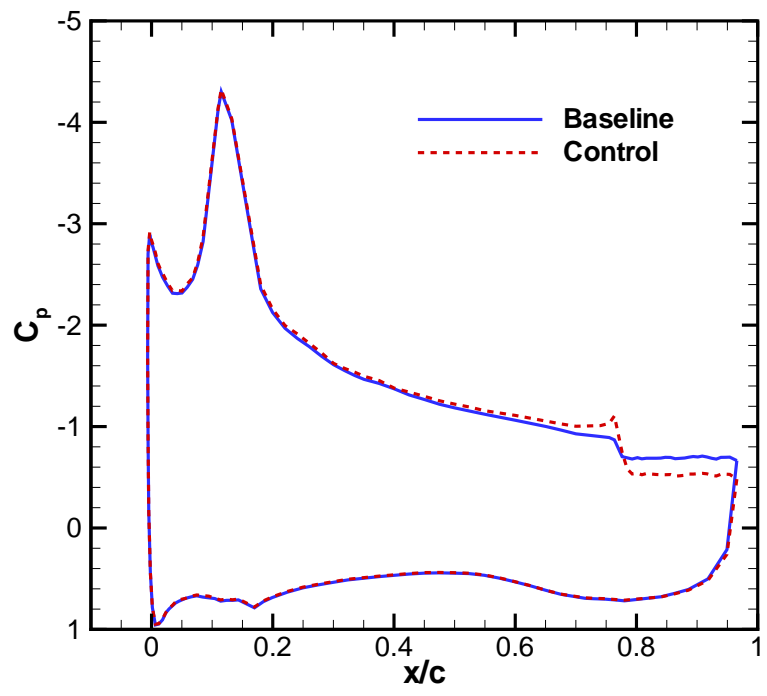

(a) $\mathrm{Re}_{\mathrm{c}}=1.7 \times 10^{6}$.

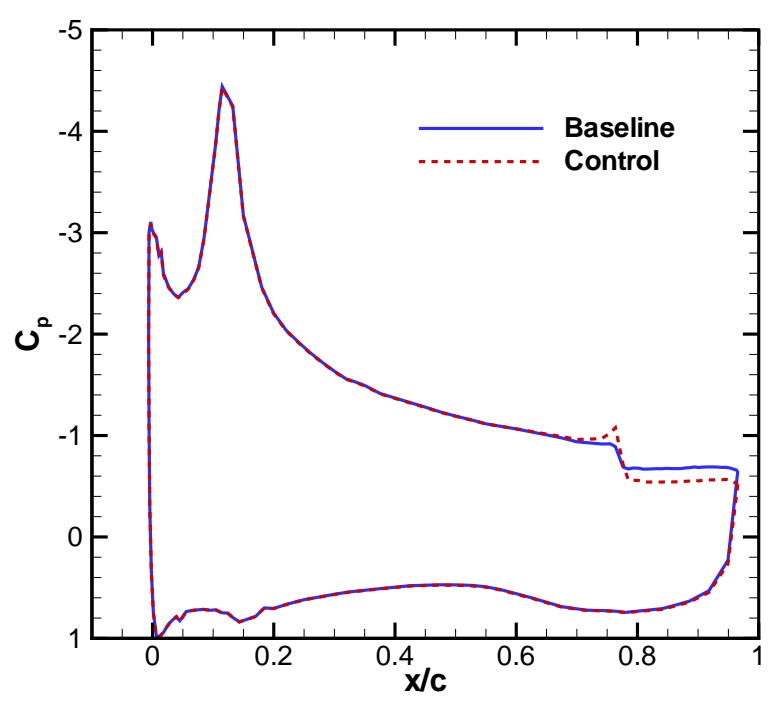

(c) $\operatorname{Re}_{\mathrm{c}}=6.0 \times 10^{6}$.

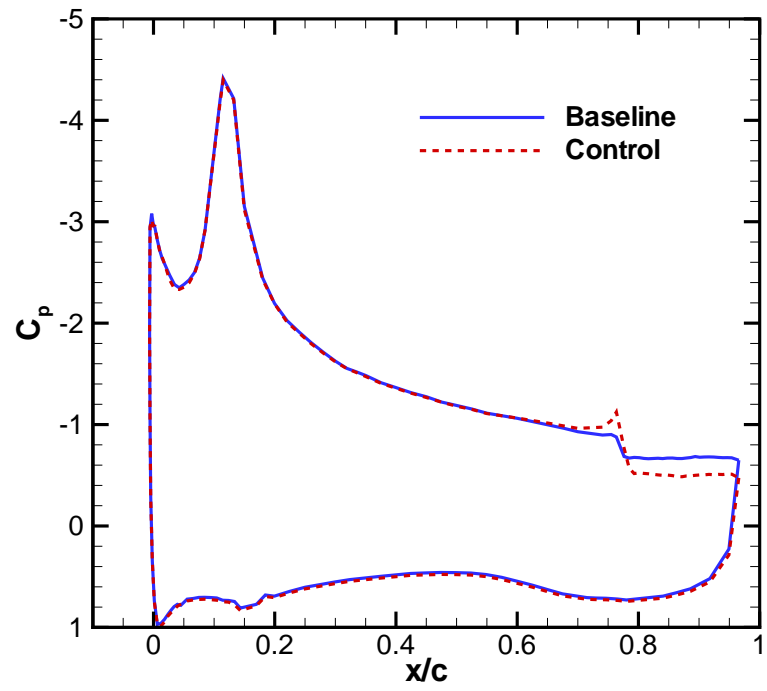

(b) $\operatorname{Re}_{\mathrm{c}}=3.4 \times 10^{6}$.

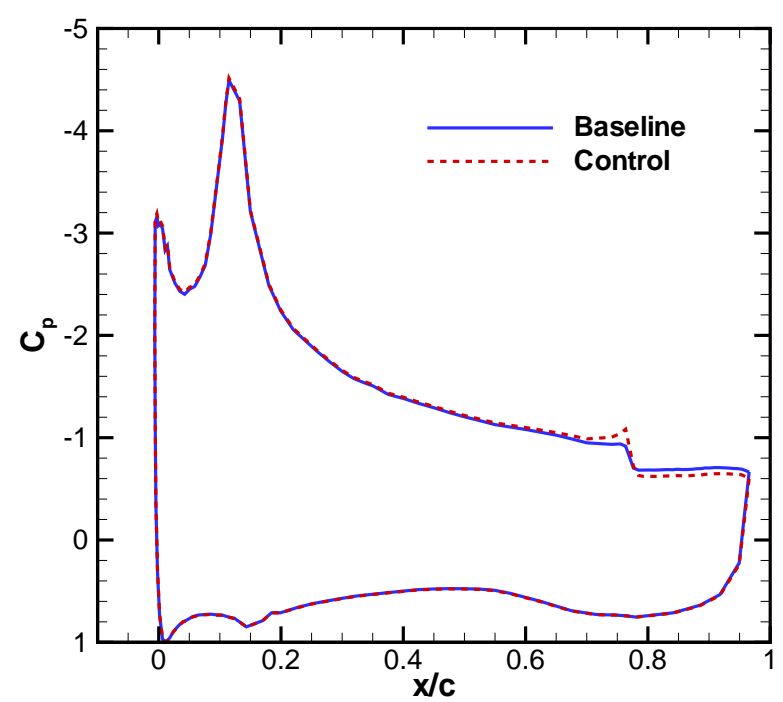

(d) $\mathbf{R e}_{\mathrm{c}}=\mathbf{9 . 0} \times 10^{6}$.

Figure 23. Reynolds number effect on TE flap actuator control. $M=0.1 ; \alpha=8^{\circ}$ (Symbols have been omitted for clarity). 


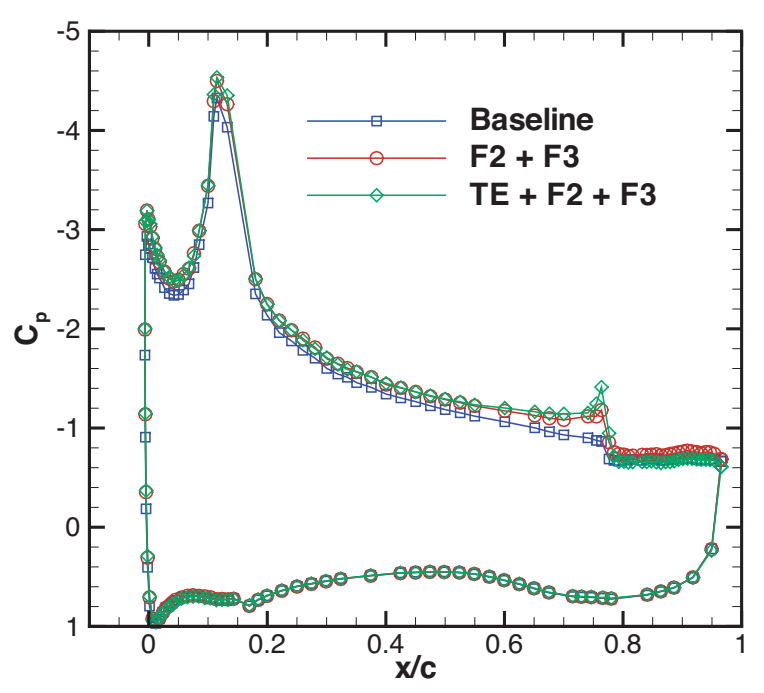

(a) $C_{p}$ distribution.

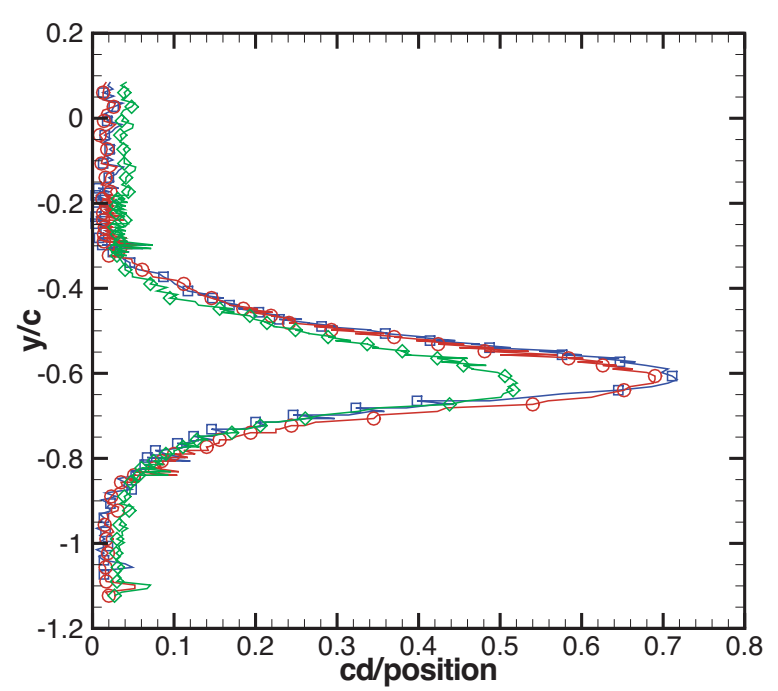

(b) Wake Profile.

Figure 24. $\mathrm{C}_{\mathrm{p}}$ and wake distributions. $\quad \alpha=8^{\circ} ; \quad \operatorname{Re}_{\mathrm{c}}=1.7 \times 10^{6} ; \quad \delta_{\mathrm{LE}}=-25^{\circ} ; \quad \delta_{\mathrm{TE}}=30^{\circ} ; \quad \mathrm{M}=0.1 ;$ $f_{\mathrm{TE}}=75 \mathrm{~Hz} ; \mathrm{f}_{\mathrm{m}, \mathrm{F} 2, \mathrm{~F} 3}=\mathbf{7 5 H z}$.

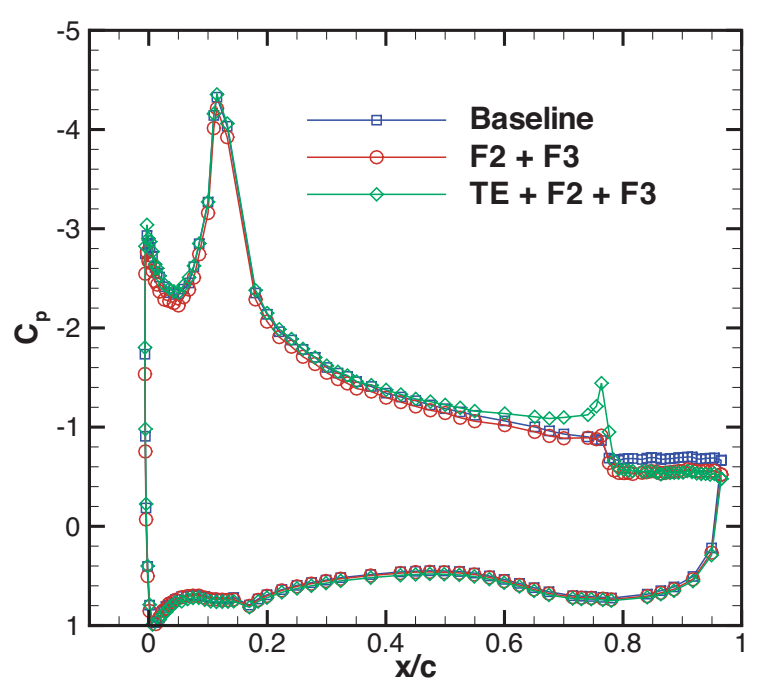

(a) $C_{p}$ distribution.

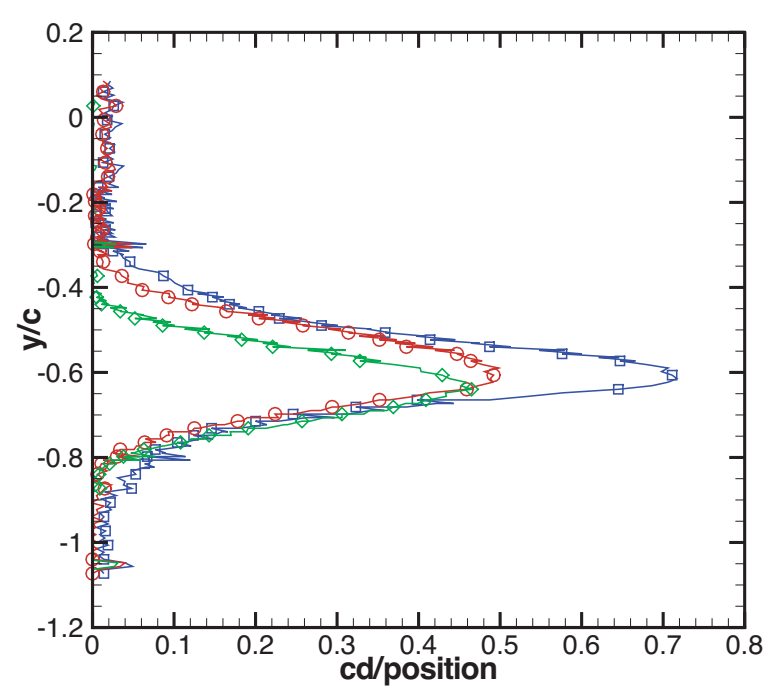

(b) Wake Profile.

Figure 25. $\mathbf{C}_{\mathbf{p}}$ and wake distributions. $\alpha=8^{\circ} ; \operatorname{Re}_{\mathrm{c}}=1.7 \times 10^{6} ; \delta_{\mathrm{LE}}=-25^{\circ} ; \delta_{\mathrm{TE}}=30^{\circ} ; \mathrm{M}=0.1 ; \mathrm{f}_{\mathrm{TE}}=150 \mathrm{~Hz} ;$ $\mathrm{f}_{\mathrm{m}, \mathrm{F} 2, \mathrm{~F} 3}=150 \mathrm{~Hz}$. 


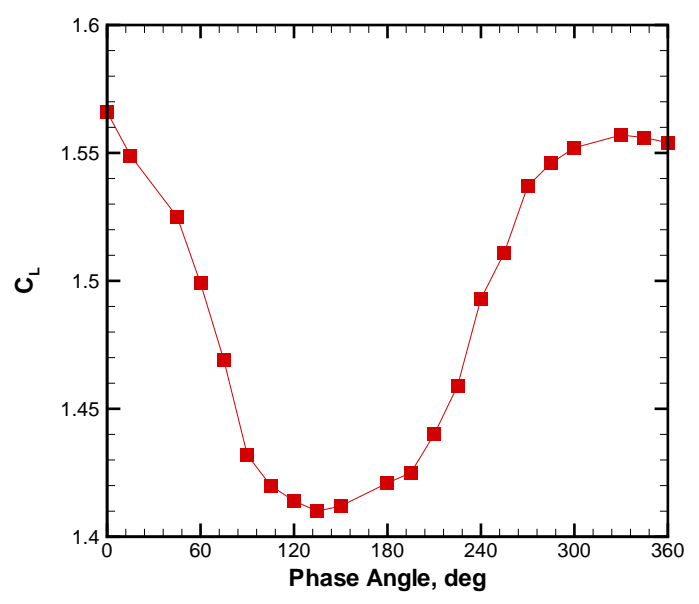

(a) $\mathbf{C}_{\mathbf{L}}$.

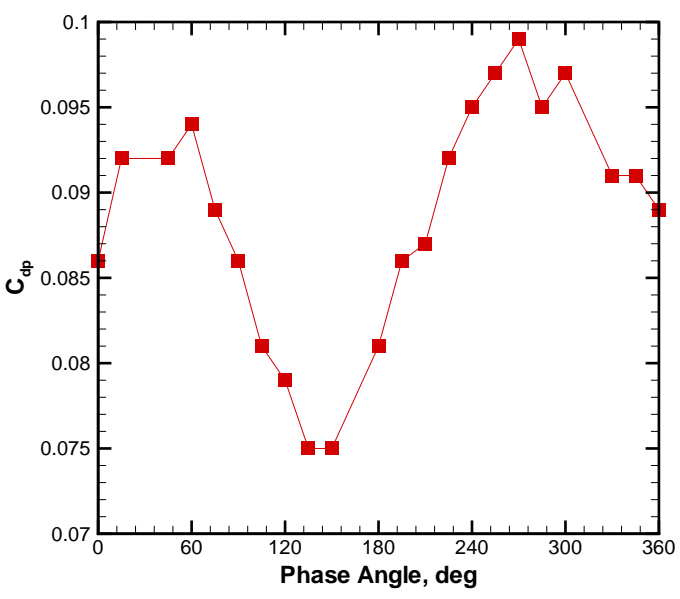

(b) $\mathbf{C}_{\mathbf{d p}}$.

Figure 26. Phase sensitivity. $\quad \alpha=0^{\circ} ; \quad \operatorname{Re}_{\mathbf{c}}=3.4 \times 10^{6} ; \quad \delta_{\mathrm{LE}}=-25^{\circ} ; \quad \delta_{\mathrm{TE}}=30^{\circ} ; \quad \mathrm{M}=0.1, \quad \mathrm{f}_{\mathrm{TE}}=150 \mathrm{~Hz} ;$ $\mathrm{f}_{\mathrm{m}, \mathrm{F2}, \mathrm{F3}}=150 \mathrm{~Hz}$.

Figure 28 presents the effect of reducing the Mach number from $\mathrm{M}=0.1$ to $\mathrm{M}=0.075$ and applying control using the TE and flap actuators. The data indicates that when the freestream velocity is reduced, thereby increasing $C_{\mu}$, the actuators around the TE flap knee of the model are more effective at controlling separation. The TE actuator increases circulation around the airfoil as well as reduces the separation region on the trailing-edge flap. The TE flap actuators, F2 and F3, are more effective at reducing $C_{d p}$.

Figure 29 presents $C_{L}$ versus angle of attack data for the airfoil with various actuators on the model active at $R e_{c}=3.4 \times 10^{6}$. The actuators are operated at maximum output and all frequencies are $\mathrm{f}=150 \mathrm{~Hz}$ and in phase. F2 and F3 are AM at $150 \mathrm{~Hz}$. This $R e_{c}$ is chosen because the flow is more 2D here because of the sidewall boundary layer removal system. The data acquired at $R e_{c}=1.7 \times 10^{6}$ is at atmospheric pressure so the sidewall boundary layer removal system is inactive due to lack of a pressure difference between the tunnel and atmosphere. At higher $R e_{c}$ values the actuator output decrease due to tunnel pressure. The data of Fig. 29 indicates that the leading edge actuator increases $C_{L, \max }$ by about $7 \%$ and delays stall by approximately $4^{\circ}$, the TE actuator increases the TE flap effectiveness increasing $C_{L}$ through a coupling with the wake and by increasing circulation, and combining the TE actuator with F2 and F3 provides an additional $C_{L}$ increment through further improvement by delaying separation, i.e. moving the separation further down the chord of the airfoil. The improvements in $C_{L, \max }$ for this airfoil configuration are similar to the results obtained by Melton et al 21

\section{Conclusions}

Zero-net-mass-flux actuators were used around the trailing-edge flap knee of a supercritical airfoil to control separation and circulation. A $0.762 \mathrm{~m}$ (30 inch) chord Energy Efficient Transport airfoil with simply hinged leading and trailing edge flaps was used for the wind tunnel experiments conducted in the NASA Langley Research Center's Low-Turbulence Pressure Tunnel. The pressurized wind tunnel enabled varying Reynolds number while holding Mach number fixed. This set of experiments was intended to extend the Reynolds number range of the database generated on the $0.4064 \mathrm{~m}$ (16 inch) chord version of the simplified high-lift EET model that had been extensively tested at lower Reynolds numbers in the NASA Langley Research Center's Basic Aerodynamics Research Tunnel. The LTPT SHL model was tested with a leadingedge flap deflection of $-25^{\circ}$ and a trailing-edge flap deflection of $30^{\circ}$ at $\mathrm{M}=0.1$ and maximum Reynolds number of $9 \times 10^{6}$. The larger LTPT model increased the available options for possible ZMF actuator 


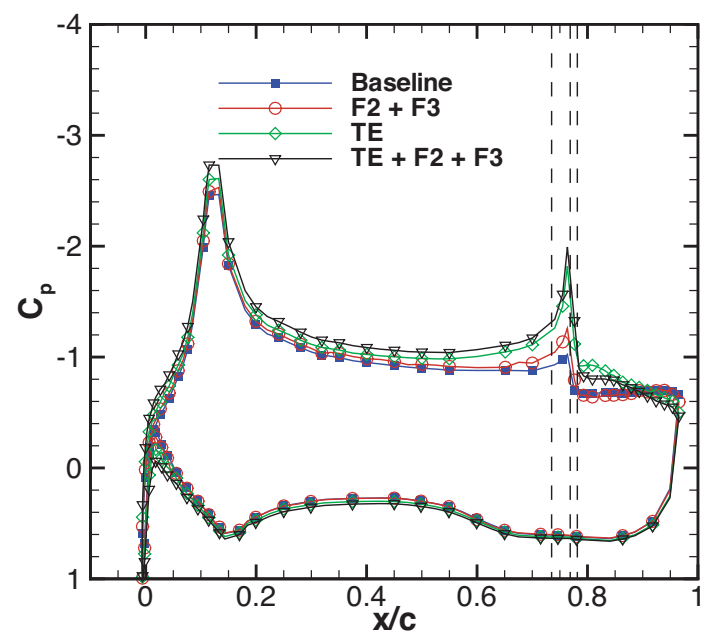

Figure 27. $\mathrm{C}_{\mathrm{p}}$ distribution. $\alpha=0^{\circ} ; \mathbf{R e}_{\mathrm{c}}=3.4 \times 10^{6}$; $\delta_{\mathrm{LE}}=-25^{\circ} ; \quad \delta_{\mathrm{TE}}=30^{\circ} ; \quad \mathrm{M}=0.1 ; \quad \mathrm{f}_{\mathrm{TE}}=150 \mathrm{~Hz}$ $\mathrm{f}_{\mathrm{F} 2}=\mathrm{f}_{\mathrm{F} 3}=150 \mathrm{~Hz}$. The vertical dashed lines represent the TE, F3, and F2 actuator slot locations.

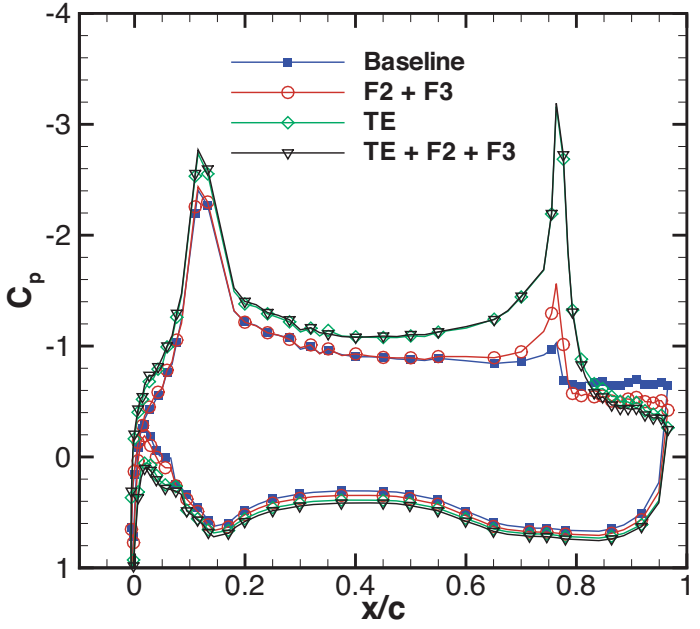

Figure 28. $\mathrm{C}_{\mathrm{p}}$ distribution. $\alpha=0^{\circ} ; \mathbf{R e}_{\mathrm{c}}=1.2 \times 10^{6}$; $\delta_{\mathrm{LE}}=-25^{\circ} ; \quad \delta_{\mathrm{TE}}=30^{\circ} ; \quad \mathrm{M}=0.075 ; \quad \mathbf{f}_{\mathrm{TE}}=150 \mathrm{~Hz} ;$ $f_{\mathrm{F} 2}=\mathbf{f}_{\mathrm{F} 3}=150 \mathrm{~Hz}$.

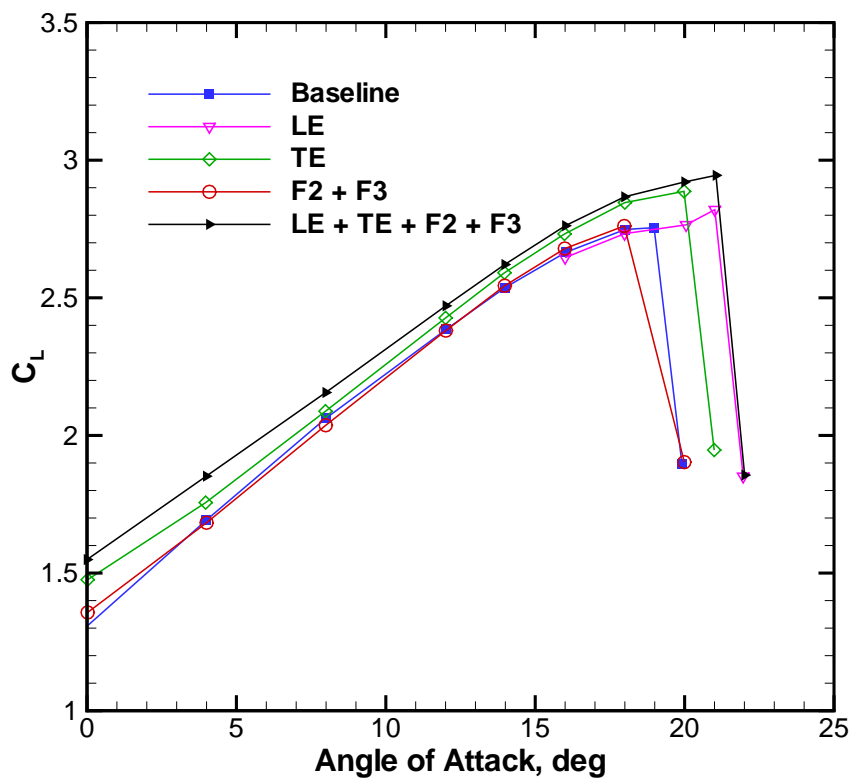

Figure 29. $\mathrm{C}_{\mathbf{L}}$ versus $\alpha$ using different actuator combinations. $\operatorname{Re}_{\mathbf{c}}=3.4 \times 10^{6} ; \mathbf{L E A}\left(\mathrm{F}^{+}=\mathbf{2 . 8 0}, \mathrm{C}_{\mu}=\mathbf{0 . 0 4} \%\right)$, TE Actuator $\left(F^{+}=0.9, C_{\mu}=0.06 \%\right)$, TE Flap Actuators $\left(F_{\mathrm{m}, \mathrm{F} 2}^{+}=0.80, \mathbf{F}_{\mathrm{m}, \mathrm{F} 3}^{+}=0.86, \mathbf{C}_{\mu}=0.06 \%\right)$. 
designs, thus providing the possibility for having more actuator control authority than was available for the BART model.

Excitation introduced upstream of the trailing-edge flap that produced reduced frequency, $F^{+}$, values in the range of 1 with $C_{\mu}$ values less than $1.0 \%$ delayed separation. Exciting the flow with an excitation frequency near the vortex shedding frequency of the airfoil, which is at a $F^{+}<1$, caused the greatest improvement in lift but was less effective at decreasing drag than the higher frequencies studied. The lift increment obtained with the control was reduced with increasing Reynolds number. This reduction in increment is due to the reduction in actuator output with increasing total pressure. The lift increment observed when operating the TE actuator near the vortex shedding frequency was reduced near $C_{L, \max }$ possibly due to the excitation frequency no longer locking with the vortex shedding frequency of the airfoil and possibly due to flow separation being upstream of the excitation slot location.

Two piezoelectric actuators on the trailing edge flap were used in combination to increase control authority. The results indicated that the actuators could be combined to control flow separation more effectively. The TE flap actuators were more effective at reducing drag than increasing lift. This was due to the fact that the actuators, when operated at resonance, primarily increased the pressures on the trailing edge flap but did not increase circulation of the airfoil. When the high frequency resonant actuators were amplitude modulated to reduced frequencies near the natural vortex shedding frequency of the airfoil, the actuators were more effective at increasing lift but the airfoil drag was also increased. The momentum required to generate a given lift increment could be reduced by amplitude modulating the high frequency excitation of the trailing edge flap actuators.

When the actuators upstream and downstream of the TE flap knee were combined, the phase sensitivity observed with the BART SHL model was confirmed although shifted by $30^{\circ}$. The current actuator arrangement produced the maximum lift when the relative phase between the TE actuator and the TE flap actuators was between $300^{\circ}$ and $360^{\circ}$. Overall, the results obtained on the LTPT SHL model were in good agreement with the low Reynolds number results from BART. Additional actuator authority is required to completely control flow separation on the model with the actuators in the current locations.

\section{Acknowledgments}

The authors would like to thank Avi Seifert, Anthony Washburn, Bill Sewall, Pam Phillips, Wendy Johnson, Jeff Bullock and Ann Bare for their support and guidance during the course of this research effort. The authors would also like to thank the Boeing Company, Nagib and Associates, and Tel Aviv University for the ZMF actuators used during the experiment.

\section{References}

\footnotetext{
${ }^{1}$ Melton, L. P., Schaeffler, N. W., Yao, C. S., and Seifert, A., "Active Control of Flow Separation from Supercritical Airfoil Leading-Edge Flap Shoulder," Journal of Aircraft, Vol. 42, No. 5, September-October 2005, pp. 1142 - 1149.

${ }^{2}$ Melton, L. P., Yao, C. S., and Seifert, A., "Application of Excitation from Multiple Locations on a Simplified High Lift System," AIAA Paper 2004-2324, 2004.

${ }^{3}$ Melton, L. P., Yao, C. S., and Seifert, A., "Active Control of Separation from the Flap of a Supercritical Airfoil," AIAA Journal, Vol. 44, No. 1, January 2006, pp. 34-40.

${ }^{4}$ Lin, J. C. and Dominik, C. J., "Parametric Investigation of a High-Lift Airfoil at High Reynolds Numbers," Journal of Aircraft, Vol. 34, No. 4, July - August 1997, pp. 485-491.

${ }^{5}$ Seifert, A., Darabi, A., and Wygnanski, I., "On the Delay of Airfoil Stall by Periodic Excitation," Journal of Aircraft, Vol. 33, No. 4, 1996, pp. 691-699.

${ }^{6}$ Seifert, A. and Pack, L. G., "Oscillatory Control of Separation at High Reynolds Numbers," AIAA Journal, Vol. 37, No. 9, 1999, pp. 1062-1071.

${ }^{7}$ Seifert, A. and Pack, L. G., "Active Flow Separation Control on Wall-Mounted Hump at High Reynolds Numbers," AIAA Journal, Vol. 40, No. 7, 2002, pp. 1363-1372.

${ }^{8}$ McClean, J. D., Crouch, J. D., Stoner, R. C., Sakurai, S., Feifel, G. E., Feifel, W. M., and Rush, H. M., "Study of the Application of Separation Control by Unsteady Excitation to Civil Transport Aircraft," NASA/CR 1999-209338, 1999.

${ }^{9}$ vonDoenhoff, A. E. and Abbott, F. T. J., "The Langley Two-Dimensional Low-Turbulence Pressure Tunnel," NACA TN 1283, National Advisory Committee for Aeronautics, 1947.

${ }^{10}$ Wlezien, R. W., Spencer, S. A., and Grubb, J. P., "Comparison of Flow Quality in Subsonic Pressure Tunnels," AIAA Paper 94-2503, 1994.

${ }^{11}$ Barlow, J. B., W. H. Rae, J., and Pope, A., Low Speed Wind Tunnel Testing, John Wiley and Son, 1999.
} 